\title{
THE ARITHMETIC PERFECTION OF BUCHSBAUM-EISENBUD VARIETIES AND GENERIC MODULES OF PROJECTIVE DIMENSION TWO BY
}

\author{
CRAIG HUNEKE
}

\begin{abstract}
We prove the ideals associated with the construction of generic complexes are prime and arithmetically perfect. This is used to construct the generic resolution for modules of projective dimension two.
\end{abstract}

Introduction. Let $R$ be a commutative Noetherian ring with identity and $I$ an ideal of $R$. If the grade of $I$ (the length of the longest $R$-sequence in $I$ ) is equal to the projective dimension of $I, I$ is said to be "perfect". The associated primes of a perfect ideal $I$ all have the same grade as $I$. If $R$ is Cohen-Macaulay, and $I$ a perfect ideal, then $R / I$ is again Cohen-Macaulay. The classical example of a perfect ideal is due to Macaulay: in a polynomial ring over a field, any ideal of height $n$ which is generated by $n$ elements is perfect.

If $R$ is regular and $R / I$ has no nontrivial idempotents, then $I$ is perfect if and only if $R / I$ is Cohen-Macaulay. In particular, if $R$ is a polynomial ring over the integers or over a field and $I$ is a homogeneous ideal this holds.

Several large classes of perfect ideals are known, especially among "determinantal" ideals. The main example is the $t \times t$ minors of a generic $r \times s$ matrix as was established by Eagon and Hochster [15] and Musili [39]. For other work, see [1], [5], [9], [13], [14], [16], [19], [21], [24], [26], [31], [34], [35], [36], and [46].

In the above papers four main methods have arisen to prove a given ideal $I$ in a polynomial ring is perfect. The first of these is the actual computation of the projective resolution of $I$ as was done in [9], [16], [19], and [37], and of which the Kozsul complex may be seen as the queen-mother. In practice this method is the most difficult; even the resolution of the maximal minors of a generic $r \times s$ matrix remains unknown over the integers; in characteristic 0 this has been solved by Lascoux [37].

The second method was realized by Hochster and Roberts in [24] where they show:

THEOREM 1.1A [24]. Let $G$ be a linearly reductive affine linear algebraic group over a field $K$ of arbitrary characteristic acting $K$-rationally on a regular Noetherian $K$-algebra $S$. Then the ring of invariants $R=S^{G}$ is Cohen-Macaulay.

Received by the editors February 16, 1980.

1980 Mathematics Subject Classification. Primary 14M12; Secondary 13H10.

Key words and phrases. Cohen-Macaulay, determinantal, complex. 
This result was later generalized by Boutot who proved

TheOREM 1.2A (Boutot [5]). Let $R$ and $S$ be essentially of finite type over a field of characteristic 0 and suppose

(1) $R$ is pure in $S$,

(2) $S$ has rational singularities.

Then $R$ has rational singularities.

These results are extremely powerful and subsume many of the previous results. However this method does have two drawbacks; firstly it only works in characteristic zero for most examples and secondly the class of perfect ideals $I$ such that $R / I$ can be represented as the invariants of a linearly reductive group is probably only a small subclass of all perfect ideals.

The third method has been recently developed by DeConcini, Eisenbud and Procesi ([12]-[14]) as an offshoot of invariant theory techniques and has the added interest of working in all characteristics. This approach consists basically of finding a new $\mathbf{Z}$-basis for the ring $\mathbf{Z}\left[X_{1}, \ldots, X_{n}\right]$ which has a "straightening law" and then representing the ideal $I$ as a free $Z$-module over this basis. Again, this method has the drawback of not working in the generality one of ten needs.

The fourth method (although in fact this was the second method chronologically) was developed by Hochster and Eagon in [15], and has since been used extensively. (See [21], [26], [34], and [35].) This technique, which Eagon and Hochster call principal radical systems, consists of finding a class of ideals, closed under suitable cuttings with hypersurfaces, whose maximal elements (under inclusion) are perfect, and along which one can descend the property of perfection. In practice, this method is the most widely applicable and handles the arithmetic case. In $\$ 2$ we give a detailed description of principal radical systems, suitably modified to fit our own specific problems.

The main purpose of this paper is to find two new classes of perfect ideals, the first of which answers queries of Kempf and Eisenbud, the second of which completes the discussion of Hochster in [22]. We now describe these questions in detail.

Let $V_{0}, \ldots, V_{n}$ be a sequence of vector spaces. (See Kempf [33] for the following discussion.) Let $V$ be the direct sum of $\operatorname{Hom}\left(V_{n}, V_{n-1}\right), \ldots, \operatorname{Hom}\left(V_{1}, V_{0}\right)$. A point in $V$ is denoted $\left(a_{n}, \ldots, a_{1}\right)$ where $a_{i}$ is an element of $\operatorname{Hom}\left(V_{i}, V_{i-1}\right)$. A point $a$ in $V$ represents a complex if $a_{i+1} a_{i}=0$ for $0<i<n$. The rank of $a$ is the sequence of integers (rank $a_{1}, \ldots$, rank $a_{n}$ ), where rank $b$ is the dimension of the image of the homomorphism $b$. If $\left(m_{n}, \ldots, m_{1}\right)$ is the rank of a complex, then $m_{n}<\operatorname{dim} V_{n}$, $m_{1} \leqslant \operatorname{dim} V_{1}$ and $m_{i}+m_{i+1} \leqslant \operatorname{dim} V_{i}$ for $0<i<n$. Conversely, any such sequence is the rank of a complex. Let $M$ be the set of such sequences.

If $m \in M$, define the variety of Buchsbaum and Eisenbud, B-E $(m)$; to be the variety of complexes $a$ such that rank $a_{i} \leqslant m_{i}$ for $1<i<n$.

In [32], Kempf showed:

TheOREM 1.3A. In characteristic $0, \mathrm{~B}-\mathrm{E}(m)$ is a normal Cohen-Macaulay variety and has rational singularities. 
Kempf states this result should hold in any characteristic and in addition, the ideal of functions vanishing on $\mathrm{B}-\mathrm{E}(\mathrm{m})$ should be generated by the quadric relations corresponding to the complex condition and the determinants corresponding to the rank conditions. We prove both of these results in $\S 6$.

THEOREM 6.0. Let $\left(a_{n}, \ldots, a_{0}\right)$ be a sequence of nonnegative integers and $\left(r_{n}, \ldots, r_{1}\right)=r$ a sequence of positive integers such that $r_{n}<a_{n}, r_{1}<a_{1}$ and $r_{i}+r_{i-1} \leqslant a_{i-1}$. Let $X_{i}$ be a generic $a_{i} \times a_{i-1}$ matrix and suppose the entries of $X_{n}, \ldots, X_{1}$ are algebraically independent. By $I_{t}\left(X_{i}\right)$ denote the ideal generated by the $t+1 \times t+1$ minors of $X_{i}$. Let $I$ be the ideal in $R=\mathbf{Z}\left[X_{n}, \ldots, X_{1}\right]$ generated by the entries of $X_{i} X_{i-1}$ for $0<i<n$ and by all the $I_{r_{i}}\left(X_{i}\right)$. Then $R / I$ is a CohenMacaulay domain and is the homogeneous coordinate ring of $\mathrm{B}-\mathrm{E}(r)$.

In fact, to show this result we need to show considerably more. The full class of perfect ideals is given by the next result.

THEOREM 6.1. Let the notation be as in Theorem 6.0. Let $s=\left(s_{0}, \ldots, s_{m}\right)$ be a strictly increasing sequence of nonnegative integers such that $s_{0}=0, s_{m}=a_{0}$ and $m \leqslant r_{1}$. Let $J$ be the ideal in $R=\mathbf{Z}\left[X_{n}, \ldots, X_{1}\right]$ generated by $I$, all $t+1 \times t+1$ minors of the first $s_{t}$ columns of $X$, and the first $s_{h}$ entries along the top row of $X_{1}$. Then $R / J$ is a Cohen-Macaulay domain. We call such a $J$ an ideal of B-E type.

This result clearly mimics the work of Eagon and Hochster in [15]. A special case of Theorem 6.1 was shown in [15]; essentially they show:

Proposition 1.1A [15]. Let $X$ be a generic $r \times s$ matrix with $r<s$ and $Y a$ generic $s \times 1$ matrix such that the entries of $X$ and $Y$ together are algebraically independent. Then if $I$ is the ideal in $\mathrm{Z}[X, Y]$ generated by the entries of $X Y, I$ is a perfect prime ideal of grade $r$.

Both of the above theorems can be extended to an arbitrary Noetherian ring using the well-known technique of generic perfection developed by Northcott, Eagon and Hochster in [17], [40], [41] and [20].

We make this extension in detail in $\$ 6$.

We do not prove the normality of the rings $R / J$, where $J$ is of B-E type in full generality although a detailed computation would provide this. Instead, we only compute the normality in the case we discuss in the second half of this paper; resolutions of length two.

The second question we address deals with the generic family of rings constructed by Hochster [22] for resolutions of length 2. We describe his result briefly.

We will say a free resolution $\mathrm{F}: 0 \rightarrow F_{2} \rightarrow F_{1} \rightarrow F_{0}$ is of type $\left(b_{2}, b_{1}, b_{0}\right)=b$ if rank $F_{i}=b_{i}$. A pair $(S, \mathbf{F})$ is said to be universal of type $b$ if $S$ is a Noetherian ring, $F$ a free $S$-resolution and if for any commutative Noetherian ring $R$ and any resolution $\mathbf{G}$ over $R$ of type $b$ with basis chosen, there exists a unique map $f: S \rightarrow R$ such that $\mathbf{G}=\mathbf{F} \otimes_{S} R$.

In [22], Hochster constructed such universal generic pairs $(S, F)$ for resolutions of length 2 . We briefly sketch his proof: let $b=\left(b_{2}, b_{1}, b_{0}\right)$ and let $X$ be a $b_{2} \times b_{1}$ 
matrix, $Y$ a $b_{1} \times b_{0}$ matrix such that the entries of $X$ and $Y$ are algebraically independent. Suppose $b_{2}<b_{1} \leqslant b_{2}+b_{0}$ and set $r=\left(b_{2}, b_{1}-b_{2}\right)$. Let $I$ be the Buchsbaum-Eisenbud ideal of type $r$ in $Z[X, Y]$ and set $R=Z[X, Y] / I$. By Theorem 6.1, $R$ is a Cohen-Macaulay normal ring and there is a complex over $R$,

$$
0 \rightarrow F_{2} \stackrel{\bar{x}}{\rightarrow} F_{1} \stackrel{\bar{Y}}{\rightarrow} F_{0}
$$

where $F_{i}$ is a free $R$-module of rank $b_{i}$ and $\bar{X}$ and $\bar{Y}$ denote the images of the matrices $X$ and $Y$ in $R$. By construction, $\operatorname{rank} \bar{X}=\operatorname{rank} F_{2}$ and $\operatorname{rank} \bar{X}+\operatorname{rank} \bar{Y}$ $=\operatorname{rank} F_{1}$.

We would like (1) to be exact. For this, there is the criteria of Buchsbaum and Eisenbud [7].

THEOREM 1.4A. Let $R$ be a commutative Noetherian ring and

$$
F: 0 \rightarrow F_{n} \stackrel{f_{n}}{\rightarrow} \cdots \stackrel{f_{1}}{\rightarrow} F_{0}
$$

a complex of free $R$-modules. Then $F$ is exact if and only if the following two conditions are satisfied:

(1) $\operatorname{rank} f_{i}+\operatorname{rank} f_{i-1}=\operatorname{rank} F_{i}$.

(2) If $I\left(f_{i}\right)=I_{\text {rank } f_{i}}\left(f_{i}\right)$ then grade $I\left(f_{i}\right) \geqslant i$.

Hence for (1) to be exact we need grade $I(\bar{Y})>1$ and grade $I(\bar{X})>2$. As $R$ is a domain the former is satisfied, but the latter condition fails for obvious reasons: the multipliers (as in Buchsbaum-Eisenbud [8]) are not in the ring $\boldsymbol{R}$.

Specifically let $\sigma$ be a $b_{2}$ subset of $\left\{1, \ldots, b_{1}\right\}$, and let $\bar{\sigma}$ be the complementary set. Then $\sigma$ determines a $b_{2} \times b_{2}$ minor of $X$ by taking the minor determined by the columns of $X$ corresponding to elements of $\sigma$. Denote this minor by $f_{\sigma}$. If $\tau$ is any $b_{1}-b_{2}$ element subset of $\left\{1, \ldots, b_{0}\right\}$ then $\bar{\sigma}$ and $\tau$ naturally determine a $\left(b_{1}-b_{2}\right)$ $\times\left(b_{1}-b_{2}\right)$ minor of $Y$ by taking rows corresponding to $\bar{\sigma}$ and columns corresponding to $\tau$. We denote this minor by $g_{\sigma, \tau}$.

Buchsbaum and Eisenbud have shown that if $R$ is a commutative Noetherian ring and $0 \rightarrow F_{2} \stackrel{X}{\rightarrow} F_{1} \stackrel{Y}{\rightarrow} F_{0}$ is an exact sequence of free $R$-modules of type $b=\left(b_{2}, b_{1}, b_{0}\right)$ then the elements $g_{\sigma, \tau} / f_{\sigma} \in R$ and $f_{\sigma} g_{\sigma^{\prime}, \tau}=f_{\sigma^{\prime}} g_{\sigma, \tau}$.

We return to our situation. Clearly the $g_{\sigma, \tau} / f_{\sigma}$ of $\bar{X}$ and $\bar{Y}$ are not in $R$ and so $I_{b_{2}}(\bar{X}) \subseteq\left(g_{\sigma, \tau}: f_{\sigma}\right)$ but $f_{\sigma} \notin R g_{\sigma, \tau}$. This shows $I_{b_{2}}(\bar{X})$ has grade 1 .

Thus, we let $S=$ the integral closure of $R\left[g_{\sigma, \tau} / f_{\sigma}\right]$ where $\sigma$ runs through all $b_{2}$-element subsets of $\left\{1, \ldots, b_{1}\right\}$ and $\tau$ runs through all $\left(b_{1}-b_{2}\right)$-element subsets of $\left\{1, \ldots, b_{0}\right\}$. Hochster shows,

Theorem 1.5A [22]. The pair $(S, \mathrm{~F})$,

$$
\text { F: } 0 \rightarrow F_{2} \stackrel{\bar{x}}{\rightarrow} F_{1} \stackrel{\bar{Y}}{\rightarrow} F_{0}
$$

is a universal generic pair of type $b=\left(b_{2}, b_{1}, b_{0}\right)$.

Hochster points out that our knowledge of modules of projective dimension two nonetheless remains incomplete; for if $S \neq R\left[g_{\sigma, \tau} / f_{\sigma}\right]$ then the integral equation for an element of $S$ not in $R\left[g_{\sigma, \tau} / f_{\sigma}\right]$ must correspond to additional structure of any free resolution of length 2 . 
The question whether $R\left[g_{\sigma, \tau} / f_{\sigma}\right]=S$ is easily seen (see $\S 7$ ) to be one of whether or not $R\left[g_{\sigma, \tau} / f_{\sigma}\right]$ satisfies Serre's condition $S_{2}$. In particular, if $R\left[g_{\sigma, \tau} / f_{\sigma}\right]$ were Cohen-Macaulay, then it would indeed be the generic ring and there would be no additional structure.

The normality of a ring of this type follows from the analytic behavior of a suitable ideal. More precisely,

Proposition 8.1. Let $R$ be a Noetherian normal domain and $I=\left(a_{1}, \ldots, a_{n}, b\right)$ an ideal such that $\operatorname{gr}_{I}(R)$ is reduced. Then $S=R\left[a_{1} / b, \ldots, a_{n} / b\right]$ is normal.

If $I$ is a height one prime and $I^{(n)}=I^{n}$ for all $n, \operatorname{gr}_{I}(R)$ is a domain and so $S=R\left[a_{1} / b, \ldots, a_{n} / b\right]$ is normal.

We apply Proposition 8.2 to the situation described above by letting $b=f_{\sigma}$ where $\sigma=(n-r+1, \ldots, n)$ and the $a_{i}$ are the $g_{\sigma, \tau}$, and $R$ is as above. The question becomes one of deciding whether or not $\left(f_{\sigma}, g_{\sigma, \tau}\right)^{(n)}=\left(f_{\sigma}, g_{\sigma, \tau}\right)^{n}$.

The literature concerning symbolic powers is extensive; for example see [23], [11], [28], [29], [12], [1], [30], [44], or [45]. There are few general methods for determining if $p^{(n)}=p^{n}$ for all $n$ given a prime $p$, but the method of weak $d$-sequences established in [28] handles most cases connected with determinantal phenomena.

Let $H$ be a finite poset, and $\left\{x_{\alpha}\right\}$ a set of elements in a commutative ring $R$ indexed by $\alpha$ in $H$. If $I$ is an ideal of $R, I$ is said to be an $H$-ideal if $I$ is generated by some subset $\left\{x_{\lambda} \mid \lambda \in \Lambda\right.$ \} of the $\left\{x_{\alpha}\right\}$ and $\Lambda$ has the property that $\beta \in \Lambda$ and $\alpha<\beta$ imply $\alpha \in \Lambda$. We set $I_{\alpha}=\left\langle x_{\beta} \mid \beta<\alpha\right\rangle$, which is clearly an $H$-ideal. If $J$ is an ideal, by $J^{*}$ we denote the ideal generated by all $x_{\beta} \in J$. We set $K=\left\langle x_{\alpha}\right| \alpha \in$ $H>$.

Definition. Let $R, H,\left\{x_{\alpha}\right\}$ be as above. We will say the $\left\{x_{\alpha}\right\}$ form a weak $d$-sequence if whenever $I$ is an $H$-ideal containing $I_{\alpha}$ but not $x_{\alpha}$ then

(1) $\left(I: x_{\alpha}\right) \cap K=\left(I: x_{\alpha}\right)^{*}$ is an $H$-ideal.

(2) If $x_{\beta} \in\left(I: x_{\alpha}\right)$ then $x_{\alpha} x_{\beta} \in I K$.

(3) If $x_{\alpha} \notin\left(I: x_{\alpha}\right)$ then $\left(I: x_{\alpha}\right)=\left(I: x_{\alpha}^{2}\right)$.

Of course, if all $\boldsymbol{H}$-ideals are self-radical, (3) will automatically hold. If $\left\{x_{\alpha}\right\}$ are a weak $d$-sequence, an ideal $J$ is said to be related to $\left\{x_{\alpha}\right\}$ if $J$ is of the form $\left(I: x_{\alpha}\right)+K$ where $I$ is an $H$-ideal containing $I_{\alpha}$ but not $x_{\alpha}$. The main result of [28] is:

THEOREM 1.5. Let $R$ be a commutative ring and $\left\{x_{\alpha} \mid \alpha \in H\right\}$ a weak $d$-sequence in $R$. Let $I$ be the ideal generated by all the $x_{\alpha}$. Then for all $n, R / I^{n}$ has a filtration $R / I^{n}=M_{0} \supseteq \ldots \supseteq M_{d}=(0)$ of $R$-modules such that $M_{k} / M_{k+1}$ is isomorphic to $R / J$ where $J$ is a related ideal of the $x_{\alpha}$.

Corollary. Suppose $R$ is a commutative ring and $\left\{x_{\alpha} \mid \alpha \in H\right\}$ a weak d-sequence. Suppose the ideal I generated by all $x_{\alpha}$ is prime and $I_{q}^{(n)}=I_{q}^{n}$ for prime ideals $q$ associated to any related ideal. Then $I^{(n)}=I^{n}$.

We show the ideal of maximal minors of the last $b_{1}-b_{2}$ rows of $Y$ and the maximal minor of $X$ determined by the last $b_{2}$ columns form a weak $d$-sequence. This is extremely easy from the principal radical system of $\$ 4$ for the BuchsbaumEisenbud ideals. Not only is this verification easy, but all the related ideals are 
prime ideals in the principal radical system and the various localizations are easy to compute.

Throughout this paper, if $K$ is a ring and $X$ a matrix with coefficients in some ring $S$ containing $K, K[X]$ will mean the subring of $S$ generated by the entries of $X$. For basic terminology and concepts such as grade or depth, we refer the reader to Matsumura [38]. All rings are commutative with one. Any result used from another paper will be denoted with an "A", i.e. Theorem 1.6A.

2. Principal radical systems. The concept of principal radical systems (PRS) was developed by Eagon and Hochster in [15]. The form has been modified both by Hochster [21] and others. For the purposes of this paper we use the following definition and lemma.

Definition 2.1 (PRS). Let $H$ be a finite poset and $x_{i}$ a set of elements in a commutative ring $R$ indexed by $i \in H$. Set $X=\left\langle x_{i}\right\rangle$. Let $W$ be a finite collection of ideals in $R$ satisfying the following properties:

If $I \in W$ and, for all $j<i, x_{j} \in I$ then $\left(I, x_{i}\right) \in W$, and there is a $J \in W$ such that $I \subseteq J \subseteq\left(I: x_{i}\right)$ and $x_{i}$ is a nonzero divisor on $R / \operatorname{rad}(J) .(\operatorname{Here} \operatorname{rad}(J)$ is the nilradical.) Further suppose that $\cap\left(I, x_{i}^{n}\right)=I$. (This automatically holds in the local or homogeneous case.) Then the collection $W$ is said to be a principal radical system (PRS).

Lemma 2.1. Let $W$ be a PRS and suppose, for all $I \in W, I+X$ is self-radical. Then

(1) All $I \in W$ are self-radical.

(2) If $I \in W$ and $x_{j} \in I$ for all $j<i$ then $\left(I: x_{i}\right) \in W$ and $\left(I: x_{i}\right)=\left(I: x_{i}^{2}\right)$.

(3) If there is an ideal $Q$ which is not contained in any associated prime of $I+X$ for any $I \in W$, then $Q$ is not contained in any associated prime of $I$ for any $I \in W$.

Proof. We prove (1) first. Let $I \in W$ be a maximal element of $W$ (under inclusion) which is not self-radical. If $X \subseteq I$, then $I=I+X$ is self-radical by assumption so we may suppose $X$ is not contained in $I$. As $H$ is finite there is a least $i \in H$ such that $x_{i} \notin I$. Then $\left(I, x_{i}\right) \in W$ and is larger than $I$ so by induction is self-radical. Further let $J \in W$ be such that $I \subseteq J \subseteq\left(I: x_{i}\right)$ and $x_{i}$ is not a zero-divisor on $R / \operatorname{rad} J$. Since $\operatorname{rad}(I) \subseteq \operatorname{rad}\left(I, x_{i}\right)=\left(I, x_{i}\right)$ we see

$$
\operatorname{rad}(I) \subseteq I+x_{i}\left(\operatorname{rad} I: x_{i}\right)
$$

Case 1. Suppose $J \neq I$. Then since $J$ is larger than $I$ and in $W, J$ is self-radical and $x_{i}$ is a nonzero divisor on $R / J$. But then $\left(\operatorname{rad} I: x_{i}\right) \subseteq\left(\operatorname{rad} J: x_{i}\right)=\operatorname{rad} J=J$ and so

$$
\operatorname{rad}(I) \subseteq I+x_{i} J \subseteq I \text { as } J \subseteq\left(I: x_{i}\right) .
$$

Case 2. Suppose $J=I$. Then $\left(\operatorname{rad}(I): x_{i}\right)=\operatorname{rad} I$ and $\operatorname{rad}(I) \subseteq I+x_{i} \operatorname{rad}(I)$. But then $(\operatorname{rad} I) \subseteq \cap\left(I, x_{i}^{n}\right)=I$. This proves (1).

For (2) we show that the ideal $J$ must equal $\left(I: x_{i}\right)$ and so $\left(I: x_{i}\right) \in W$. Since $J$ is self-radical, $x_{i}$ is not a zero-divisor on $R / J$. But if $y \in\left(I: x_{i}\right)$ then $x_{i} y \in I \subseteq J$ and so $y \in J$. This establishes our claim, as $x_{i}$ is not a zero-divisor on $R / J$. 
Finally we prove (3). Let $I$ be a maximal ideal (under inclusion) such that $Q$ is contained in an associated prime $P$ of $I$. If $X \subseteq I$ this contradicts the assumption so that there is a least $i$ in $H$ such that $x_{i} \notin I$.

Case $1 . I \neq\left(I: x_{i}\right)$. Then since $\left(I: x_{i}\right)=\left(I: x_{i}^{2}\right)$ by step 2 , we obtain

$$
I=\left(I, x_{i}\right) \cap\left(I: x_{i}\right) \text {. }
$$

Each of these ideals is self-radical and both the ideals on the right are strictly larger than $I$. Since no prime in either of their decompositions contains $Q$, the same holds for $I$.

Case 2. $I=\left(I: x_{i}\right)$. We may assume there is a $c \in R$ such that $c \notin I$ but $c Q \subseteq I$. Then $c Q \subseteq\left(I, x_{i}\right)$ shows $c \in\left(I, x_{i}\right)$ by the choice of $I$. Hence $c=i_{1}+c_{1} x_{i}$ and $c_{1} x_{i} Q \subseteq I$. Since $\left(I: x_{i}\right)=I$, this shows $c_{1} Q \subseteq I$ and so $I+(I: Q) x_{i}=(I: Q)$. However this implies

$$
(I: Q) \subseteq \bigcap_{n=1}^{\infty}\left(I, x_{i}^{n}\right)
$$

and so $(I: Q)=I$ which shows $Q$ is not contained in $P$.

It is worth noting the hard step in this process. This consists firstly of finding a large enough class of ideals to contain all the necessary ones and secondly proving that $x_{i}$ is not a zero-divisor on $R / \operatorname{rad}(J)$. This is usually done by finding a "generic" point for such $J$, that is a mapping of $R / J$ into a domain $D$ such that the kernel of the mapping has the same radical as $J$ and such that $x_{i}$ does not go to zero.

Principal radical systems are useful not only to prove the primeness of a given ideal but also are useful in proving the perfection of the prime ideals they contain. One can descend the property of perfection along the PRS using various easy lemmas from homological algebra as was done in [3] and [15]. We list the necessary lemmas here. (See [2].)

Lemma 2.2A. If $I$ and $J$ are perfect ideals of grade $g$ in a Noetherian ring $R$, and $I+J$ has grade $g+1$, then $I+J$ is perfect if and only if $I \cap J$ is perfect (necessarily of grade $\mathrm{g}$ ).

Lemma 2.3A [21]. Let $I_{1}, \ldots, I_{k}$ be perfect ideals of grade $g$ in a Noetherian ring $R$ and suppose for any $i, 1<i \leqslant k$, the sum of any $i$ of these ideals has grade $(g-1)+i$. Suppose also that the least family of ideals generated from $I_{1}, \ldots, I_{k}$ under the operations,$+ \cap$ is a distributive lattice under,$+ \cap$. Then the following two conditions are equivalent:

(1) The sum of every subset of the ideals $I_{1}, \ldots, I_{k}$ is perfect.

(2) The intersection of every subset of the ideals $I_{1}, \ldots, I_{k}$ is perfect.

The usefulness of this comes from the following observation.

LEMMA 2.4. Let $W$ be a PRS and suppose

(1) If $I \in W$ then $(I, X)$ is self-radical.

(2) If $I, J \in W$ both $I+J$ and $I \cap J \in W$.

Then the ideals in $W$ form a distributive lattice under,$+ \cap$. 
Proof. It is enough to observe that if $I_{1}, \ldots, I_{k}, J \in W$ then the ideals $\left(I_{1} \cap \ldots \cap I_{k}\right)+J$ and $\left(I_{1}+J\right) \cap \ldots \cap\left(I_{k}+J\right)$ have the same radical and are both in $W$. By Lemma 2.1, these ideals are self-radical and hence are equal.

3. Buchsbaum-Eisenbud varieties. In this section we begin the proof of the perfection of the Buchsbaum-Eisenbud ideals by embedding them in a suitably large principal radical system. The argument will consist of three steps: first the definition of the principal radical system and the definition of the ideal $J$ required (as in Definition 2.1), second the description of the generic points, and finally the calculation of the grade of the ideals in the principal radical system (by finding new generic points) and the application of Lemma 2.3A to descend the property of perfection.

We now fix positive integers $\left(a_{n}, \ldots, a_{0}\right)=a$ and nonnegative integers $r=$ $\left(r_{n}, \ldots, r_{1}\right)$ such that $r_{n} \leqslant a_{n}, r_{1} \leqslant a_{1}$ and $r_{i}+r_{i-1} \leqslant a_{i}$ for $1<i<n$. Fix matrices $X_{n}, \ldots, X_{1}$ with algebraically independent entries over a field $K$ (which we fix), $X_{i}$ of size $a_{i} \times a_{i-1}$ for $1<i<n$. If $X$ is a matrix we write $(X \mid i)$ to denote the first (left-most) $i$ columns of $X$. We will write $(i \mid X)$ to denote the matrix formed from the bottom $i$ rows of $X$ and throughout $I_{t}(X)$ will denote the ideal generated by all the $t+1$ by $t+1$ minors of $X$. The entries of $X_{1}$ we denote simply by $x_{i j}$; the entries of $X_{k}$ for $k>1$ we denote by $x_{i j}^{(k)}$.

For the purposes of the second problem we wish to consider in this paper two different principal radical systems will be considered simultaneously. We fix for the remainder of the discussion two ideals contained in $R=K\left[X_{n}, \ldots, X_{1}\right]$. Define

$$
L_{1}=\sum_{i=1}^{n} I_{r_{i}}\left(X_{i}\right)+\sum_{i=1}^{n} I_{1}\left(X_{i} X_{i-1}\right)
$$

and define $L_{2}=L_{1}+R \Delta$ where $\Delta$ is the $a_{n} \times a_{n}$ minor of $X_{n}$ given by the last $a_{n}$ rows of $X_{n}$. (Notice if $r_{n}<a_{n}$ then $\Delta \in L_{1}$.)

We now consider additional nonnegative integers $s=\left(s_{0}, \ldots, s_{m}\right), 0=s_{0}$ $<\ldots<s_{m}=a_{0}$ where $m \leqslant r_{1}$. We let $T$ be the set of such sequences and if $s \in T$ and $p$ is an integer $0 \leqslant p \leqslant a_{0}$ we define

$$
I(a, r, s, p)=L_{1}+\sum_{t=0}^{m} I_{t}\left(x_{1} \mid s_{t}\right)+R\left(x_{11}, \ldots, x_{1 p}\right)
$$

and define

$$
I^{2}(a, r, s, p)=L_{2}+\sum_{t=0}^{m} I_{t}\left(x_{1} \mid s_{t}\right)+R\left(x_{11}, \ldots, x_{1 p}\right) .
$$

Set $W=\left\{I(a, r, s, p) \mid s \in T\right.$ and $\left.0 \leqslant p<a_{0}\right\}$ and $W^{2}=\left\{I^{2}(a, r, s, p) \mid s \in T\right.$ and $\left.0 \leqslant p \leqslant a_{0}\right\}$.

We will show both $W$ and $W^{2}$ are principal radical systems. For the partially ordered set $H$ belonging to the principal radical system we take $x_{11}, \ldots, x_{1 s}$ ordered linearly by $x_{11}<\ldots<x_{1 s}$.

To show $W$ and $W^{2}$ are principal radical systems we first observe that all the ideals are homogeneous and hence the condition $\cap\left(I, x_{i}^{n}\right)=I$ is immediate. If $I(a, r, s, p) \in W$ then $\left(I(a, r, s, p), x_{1 p+1}\right)=I(a, r, s, p+1) \in W$. Finally suppose 
$I(a, r, s, p) \in W$ and $s_{h} \leqslant p<s_{h+1}$. We define an $s^{\prime} \in T, s^{\prime}=\left\{s_{0}^{\prime}, \ldots, s_{m}^{\prime}\right\}$ where $s=\left\{s_{0}, \ldots, s_{m}\right\}$ by

$$
\begin{aligned}
& s_{j}^{\prime}=s_{j} \quad \text { if } j \neq h, \\
& s_{h}^{\prime}=p .
\end{aligned}
$$

Clearly $I(a, r, s, p) \subseteq I\left(a, r, s^{\prime}, p\right)$. We will show $I\left(a, r, s^{\prime}, p\right)$ satisfies the conditions necessary for the ideal $J$ of Definition 2.1.

For the remainder of this discussion since $a=\left(a_{n}, \ldots, a_{0}\right)$ and $r=\left(r_{n}, \ldots, r_{1}\right)$ are fixed, we let $I(a, r, s, p)=I(s, p)$.

LeMma 3.1. Let the notation be as above. Then $I\left(s^{\prime}, p\right) \subseteq\left(I(s, p): x_{1 p+1}\right)$.

Proof. From the definition of $s^{\prime}$, it is clear we need only show that if $\Delta$ is any $h+1 \times h+1$ minor of the first $p$ rows of $X_{1}$, then $x_{1 p+1} \Delta \in I(s, p) . \Delta$ is determined by $h+1$ rows $R$ and $h+1$ columns $C$ of $X_{1}$. If $\{1\} \subset R$, then $\Delta \in I(s, p)$. If we let $R^{\prime}=\{1\} \cup R$ and $C^{\prime}=\{p+1\} \cup C$ then the sets $R^{\prime}$ and $C^{\prime}$ determine an $h+2 \times h+2$ minor $W$ of the first $p+1$ rows. Since $p<s_{h+1}$, $W \in I(s, p)$. If we expand $W$ along the first row, $W=x_{1 p+1} \Delta+W^{\prime}$ where $W^{\prime} \in\left(x_{11}, \ldots, x_{1 p}\right)$. Since this shows $W^{\prime} \in I(s, p)$ we see $x_{1 p+1} \Delta \in I(s, p)$ as required.

Consequently, to show $W$ is a principal radical system it is enough to show the radicals of the various $I(s, p)$ do not have irreducible components containing $x_{1 p+1}$. For this we construct generic points for ideals $I(s, p) \in W$ such that $p=s_{h}$ for some $s_{h}$.

4. Generic points for $I(s, p)$. In this section we use the notation established in [15]. If $X, X^{\prime}$ are $r$ by $s$ and $r$ by $s^{\prime}$ matrices respectively, we let $X \# X^{\prime}$ be the $r$ by $s+s^{\prime}$ matrix formed by juxtaposing $X$ and $X^{\prime}$. If $Y$ is an $r^{\prime}$ by $r$ matrix clearly

$$
Y\left(X \# X^{\prime}\right)=(Y X) \#\left(Y X^{\prime}\right) \text {. }
$$

If $X$ is a matrix and $K$ a ring we let $K[X]$ represent the ring $K$ with the entries of $X$ adjoined.

As in $\$ 3$ we fix positive integers $a=\left(a_{n}, \ldots, a_{0}\right)$ and nonnegative integers $r=\left(r_{n}, \ldots, r_{1}\right)$ such that $r_{n} \leqslant a_{n}, r_{1} \leqslant a_{1}$ and $r_{i}+r_{i-1} \leqslant a_{i-1}$ for $1<i<n$. We let $X_{n}, \ldots, X_{1}=X$ be matrices whose entries are algebraically independent over a field $K$ and such that $X_{i}$ is $r_{i} \times r_{i-1}$. As in $\S 3$, we let $s \in T$ and $0<p \leqslant a_{0}$ such that $p=s_{h}$ for some $s_{h}$ in $H$.

Now let $A_{k}$ be an $r_{k}$ by $r_{k}(2<k<n-1)$ matrix with indeterminate coefficients; in the future we always assume the entries of all such matrices taken together are algebraically independent. Let $A_{n}$ be an $a_{n}$ by $r_{n}$ matrix of indeterminates.

If $2 \leqslant k \leqslant n$ we let $B_{k}$ be the $a_{k}$ by $a_{k-1}$ matrix with $A_{k}$ in the top right-hand corner and zeroes elsewhere. As $r_{k} \leqslant a_{k}$ and $r_{k} \leqslant a_{k-1}$, this is possible.

Let $E_{k}$ be an $a_{k}$ by $a_{k}$ generic matrix for $1<k \leqslant n$.

Now set $j_{k}=s_{k}-s_{k-1}(1 \leqslant k \leqslant m)$ where $s=\left(s_{0}, \ldots, s_{m}\right)$. For each $k$ in $\langle 1, m-1\rangle$ (by which we mean $\{1, \ldots, m-1\}$ ) let $U_{k}$ be a $k+1$ by $k$ matrix of indeterminates and let $U_{m}$ be an $a_{1}-r$ by $m$ matrix of indeterminates: For $k$ in 
$\langle 1, m\rangle$ let $V_{k}$ be a $k$ by $j_{k}$ matrix of indeterminates. Let $W^{\prime}$ be the $a_{1}-r_{2}$ by $s$ matrix $\left(U_{m} \ldots U_{1} V_{1}\right) \# \ldots \#\left(U_{m} \ldots U_{k} V_{k}\right) \# \ldots \#\left(U_{m} V_{m}\right)$. Let $W$ be the $a_{1} \times$ $a_{0}$ matrix with $W^{\prime}$ in its top $a_{1}-r$ rows and zeroes elsewhere. Let $Y$ be the first row of $U_{m}$ and let $P=I_{0}\left(Y U_{m-1} \ldots U_{n}\right)$. We note the following proposition found in [15].

Proposition 4.1A. Let $K$ be a Noetherian domain. Let $r=t_{m+1}>t_{m}>\ldots>t_{1}$ be a strictly decreasing sequence of positive integers. Let $U_{i}$ be $a t_{i+1}$ by $t_{i}$ matrix of indeterminates over $K, 1 \leqslant i \leqslant m$. Let $Y$ be a 1 by $r$ matrix of indeterminates. Then the entries of the 1 by $t_{1}$ product matrix $Y U_{m} \ldots U_{1}$ form an $R$-sequence in the polynomial ring generated over $K$ by the indeterminates in $Y$ and the $U_{i}^{\prime}$ 's, and the ideal generated by the entries is prime.

Let $L$ be the ring, $K\left[E_{1}, \ldots, E_{n}\right.$, $\left.\left(\operatorname{det} E_{1}\right)^{-1}, \ldots,\left(\operatorname{det} E_{n}\right)^{-1}\right]$ and define

$$
D(s, p)=L\left[B_{2}, \ldots, B_{n}, U_{m}, \ldots, U_{1}, V_{m}, \ldots, V_{1}\right] / P \text {. }
$$

By the above proposition $D(s, p)$ is a domain.

We let $R=K\left[X_{n}, \ldots, X_{1}\right]$ and define a map $f(s, p): R \rightarrow D(s, p)$ by sending the entries of $X_{n}$ to the entries of $B_{n} E_{n}^{-1}$, the entries of $X_{j}$ for $1<j<n$ to the entries of $E_{j+1} B_{j} E_{j}^{-1}$ and the entries of $X_{1}$ to the entries of $E_{1} W$.

Proposition 4.1. The map $f(s, p)$ defined above is a generic point for $I(s, p)$; that is $\operatorname{ker}(f(s, p))$ is a prime ideal and $\operatorname{rad}(I(s, p))=\operatorname{ker}(f(s, p))$. Further $x_{1 p+1}$ is not a zero-divisor on $R / \operatorname{ker}(f(s, p))$.

Proof. The last statement follows from the first since $f(s, p)\left(x_{1 p+1}\right) \neq 0$.

Let us first show that $I(s, p) \subseteq \operatorname{ker}(f(s, p))$. First, we notice that $B_{j+1} B_{j}=0$ for $1 \leqslant j \leqslant n-1$ since $r_{i}+r_{i-1} \leqslant a_{i-1}$. Hence

$$
\left(E_{j+2} B_{j+1} E_{j+1}^{-1}\right)\left(E_{j+1} B_{j} E_{j}^{-1}\right)=0 \quad \text { (where } E_{n+1}=E_{0}=\text { the identity) }
$$

and so $f(s, p)\left(X_{j+1} X_{j}\right)=0$.

Next, clearly $I_{r_{j}}\left(B_{j}\right)=0$ and so $I_{r_{j}}\left(E_{j+1} B_{j} E_{j}^{-1}\right)=0$ which shows

$$
I_{r_{j}}\left(X_{j}\right) \subseteq \operatorname{ker} f(s, p) \text {. }
$$

Finally, as $I_{t}(W \mid j)=I_{t}\left(W^{\prime} \mid j\right)$ we only need to see that $I_{t}\left(W \mid s_{t}\right)=0$.

However, $I_{t}\left(W \mid s_{t}\right)$ is just $\left(U_{m} \ldots U_{1} V_{1}\right) \# \ldots \#\left(U_{m} \ldots U_{t} V_{t}\right)=U^{*} V^{*}$ where $U^{*}=\left(U_{m} \ldots U_{t}\right)$ is $a_{1}-r$ by $t$ and $V^{*}=\left(U_{t-1} \ldots U_{1} V_{1}\right) \# \ldots \# V_{t}$ is $t$ by $\sum_{i=0}^{t}\left(s_{i}-s_{i-1}\right)=s_{t}$. Hence $I_{t}\left(W \mid s_{t}\right)=I_{t}\left(U^{*} V^{*}\right)$ and

$$
I_{t}\left(U^{*} V^{*}\right) \subseteq I_{t}\left(U^{*}\right) I_{t}\left(V^{*}\right)=0 .
$$

It remains to show $\operatorname{ker}(f(s, p)) \subseteq \operatorname{rad}(I(s, p))$. Let “-” denote the images of elements of $R$ modulo some prime $P \supset \operatorname{rad}(I(s, p))$. Let $D=K\left[X_{n}, \ldots, X_{1}\right] / P$ and let $L$ be the fraction field of $D$. It will suffice to show there exist matrices $B_{j}, E_{j}, U_{j}, V_{j}$ as above with coefficients in $L$ such that each $\bar{X}_{j}$ can be factorized into the corresponding matrices.

LeMma 4.1. Suppose $X$ and $Y$ are matrices with coefficients in a field of sizes $r \times n$ and $n \times s$ respectively and $X Y=0$. Suppose rank $X \leqslant a$ and $\operatorname{rank} Y<b$. Then there is an elementary matrix $E$ such that $X E^{-1}$ has zero entries in the first $r-a$ columns and such that $E^{-1} Y$ is zero in the last $n-b$ rows. 
Proof. This is trivial using elementary column operations (in $X$ ) and elementary row operations (on $Y$ ).

We return to the proof of Proposition 4.1. First we apply Lemma 4.1 with $X=X_{n}, Y=X_{n-1}, a=r_{n}$ and $b=r_{n-1}$. Over $L$, there exists an elementary matrix $E_{n}^{\prime}$ such that $\bar{X}_{n} E_{n}^{\prime}$ has zero entries in the first $a_{n}-r_{n}$ columns, and $\left(E_{n}^{\prime}\right)^{-1} Y$ is zero in the last $a_{n-1}-r_{n-1}$ rows. Thus there is an $a_{n}$ by $r_{n}$ matrix $A_{n}^{\prime}$ such that $\bar{X}_{n}=\left(0 \# A_{n}^{\prime}\right)\left(E_{n}^{\prime}\right)^{-1}$ where 0 is an $a_{n}$ by $a_{n}-r_{n}$ matrix with zero entries.

One may choose $E$ in such a way that the row space of $\left(t \mid X_{n-1} E^{-1}\right)$ is the same as that of $(t \mid X)$.

We now apply Lemma 4.1 to the matrix $\left(E_{n}^{\prime}\right)^{-1} \bar{X}_{n-1}$ and $\bar{X}_{n-2}$. Since $I(s, p) \subseteq P$, rank $\bar{X}_{n-1} \leqslant r_{n-1}$, and rank $\bar{X}_{n-2} \leqslant r_{n-2}$ so there is an elementary matrix $E_{n-1}^{\prime}$ over $L$ such that $B_{n-1}^{\prime}=\left(E_{n}^{\prime}\right)^{-1} \bar{X}_{n-1} E_{n-1}^{\prime}$ is zero in the first $a_{n-2}-r_{n-1}$ columns, and such that the row spaces of $B_{n-1}^{\prime}$ are the same as those of $\left(E_{n}^{\prime}\right)^{-1} \bar{X}_{n-1}$. In particular, the last $a_{n-1}-r_{n-1}$ rows of $B_{n-1}^{\prime}$ are zero. But then there is an $r_{n-1}$ by $r_{n-1}$ matrix $A_{n-1}^{\prime}$ such that $B_{n-1}^{\prime}$ is the $a_{n-1}$ by $a_{n-2}$ matrix with zeroes everywhere but in the upper right-hand corner, where $A_{n-1}^{\prime}$ is placed. Hence

$$
\bar{X}_{n-1}=E_{n}^{\prime} B_{n-1}^{\prime}\left(E_{n-1}^{\prime}\right)^{-1}
$$

has the necessary factorization. It is clear we may inductively choose the $E_{j}^{\prime}$ and $B_{j}^{\prime}$ with the proper form so that $\bar{X}_{j}=E_{j+1}^{\prime} B_{j}^{\prime}\left(E_{j}^{\prime}\right)^{-1}$ for $2<j<n-1$ and such that $E_{1}^{\prime} \bar{X}_{1}$ is zero in the last $a_{1}-r_{1}$ rows. Since $E_{1}^{\prime}$ does not change the column ranks of $\bar{X}_{1}$, the matrix $Z=E_{1}^{\prime} \bar{X}_{1}$ is such that $I_{t}\left(Z \mid s_{t}\right)=0$ since $I_{t}\left(\bar{X}_{1} \mid s_{t}\right)=0$. Further if $Z=\left(z_{i j}\right)$ we see $z_{11}=\ldots=z_{1 p}=0$. But now the necessary factorization of $E_{1}^{\prime} \bar{X}_{1}$ follows from Proposition 36 of [15], which we state below.

Proposition 4.2A [15]. Let $K$ be a Noetherian domain, $X$ an $r$ by $s$ matrix of indeterminates over $K$ and $R=U[X]$. Let $s=\left(s_{0}, \ldots, s_{m}\right)$ where $s_{0}=0$ and $s_{m}=s$. For $1 \leqslant k \leqslant m-1$ let $U_{k}$ be $a(k+1)$ by $k$ matrix of indeterminates over $K$ and let $U_{m}$ be an $r \times(m-1)$ matrix of indeterminates. For each $k, 1<k<m$, let $V_{k}$ be a $k$ by $\left(s_{k}-s_{k-1}\right)$ matrix of indeterminates and suppose all the entries of the $U$ 's and $V$ 's taken together are algebraically independent over $K$. Let $W$ be the $r$ by $s$ matrix

$$
\left(U_{m} \ldots U_{1} V_{1}\right) \# \ldots \#\left(U_{m} \ldots U_{k} V_{k}\right) \# \ldots \#\left(U_{m} V_{m}\right) \text {. }
$$

Let $P$ be the ideal given by $I_{0}\left(Y U_{m-1} \ldots U_{k}\right)$ where $Y$ is the first row of $U_{m}$. Then the $K$-homomorphism $\phi$ mapping $K[X]$ onto $K[W] / P$ by taking the entries of $X$ to the corresponding entries of $W$ is a generic point for the ideal $J_{s, p}$ which is equal to $\sum_{t=1}^{m} I_{t}\left(x \mid s_{t}\right)+\left(x_{11}, \ldots, x_{1 p}\right)$.

COROLlARY 4.1. $W$ is a principal radical system.

Proof. By Lemma 3.1 the ideal $I\left(s^{\prime}, p\right)$ satisfies

$$
I(s, p) \subseteq I\left(s^{\prime}, p\right) \subseteq\left(I(s, p): x_{1 p+1}\right)
$$

and $p=s_{h}^{\prime}$ for some $s_{h}^{\prime}$ in $s$. By Proposition $4.1, \operatorname{ker}\left(f\left(s^{\prime}, p\right)\right)$ is a generic point for $I\left(s^{\prime}, p\right)$ and $x_{1 p+1}$ is not in $\operatorname{ker}\left(f\left(s^{\prime}, p\right)\right)$. All the conditions of Definition 2.1 are now satisfied. 
It is easy to modify the matrix $E_{n}$ so that we obtain generic points for $I^{2}(s, p)$. Thus,

COROllary 4.3. $W^{2}$ is a principal radical system and if $s$ and $p$ are such that $p=s_{h}$ for some $s_{h}$ in $H$, then $\operatorname{rad}\left(I^{2}(s, p)\right)$ is prime.

TheOREM 4.1. Let $\left(a_{n}, \ldots, a_{0}\right)=a$ be any set of positive integers and $\left(r_{n}, \ldots, r_{1}\right)$ $=r$ any set of nonnegative integers such that $r_{n}<a_{n}, r_{1}<a_{0}$ and $r_{i}+r_{i-1}<a_{i-1}$. Further let $0=s_{0}<\ldots<s_{m}=a_{0}$ be integers, $s=\left(s_{0}, \ldots, s_{m}\right)$ such that $m<r_{1}$ and let $p$ be an integer, $0 \leqslant p \leqslant a_{0}$. Let $X_{i}$ be $a_{i}$ by $a_{i-1}$ matrices, $1<i<n$, and let $K$ be any field. Let $R=K\left[X_{n}, \ldots, X_{1}\right]$ and let $I(a, r, s, p)$ be the ideal in $R$ defined by

$$
\begin{aligned}
I(a, r, s, p)= & \sum_{i=1}^{n} I_{r_{i}}\left(X_{i}\right)+\left(\text { the entries of } X_{i} X_{i-1}\right) \\
& +\sum_{t=0}^{m} I_{t}\left(X_{1} \mid s_{t}\right)+\left(x_{11}, \ldots, x_{1 p}\right) .
\end{aligned}
$$

Then $I(a, r, s, p)$ is self-radical and if $p=s_{h}$ for some $0<h<m, I(a, r, s, p)$ is prime.

Proof. We induct on $\sum_{i=0}^{n} a_{i}=\tilde{a}$. By Proposition 3.1, for fixed $a$ and fixed $r$, the ideals $I(a, r, s, p)$ form a principal radical system and so by Lemma 2.1 to show they are self-radical it is enough to show that, for each $I(a, r, s, p)$, $\left(I(a, r, s, p), x_{11}, \ldots, x_{1 a_{0}}\right)$ is self-radical.

However the ideal above is of the form $I\left(a^{\prime}, r^{\prime}, s^{\prime}, 0\right)+\left(x_{11}, \ldots, x_{1 a_{0}}\right)$ where $\left(a_{n}, \ldots, a_{2}, a_{1}^{\prime}, a_{0}\right)=a^{\prime}$ and $r^{\prime}=\left(r_{n}, \ldots, r_{2}, r_{1}^{\prime}\right)$ where $a_{1}^{\prime}=a_{1}-1$ and $r_{1}^{\prime}=r_{1}$ unless $r_{1}=a_{1}$ in which case $r_{1}^{\prime}=a_{1}^{\prime}$ and where $s^{\prime}$ is suitably adjusted. As $\tilde{a}^{\prime}<\tilde{a}$ we may conclude $I\left(a^{\prime}, r^{\prime}, s^{\prime}, 0\right)$ is self-radical and as $x_{11}, \ldots, x_{1 a_{0}}$ are algebraically independent modulo this ideal we see $\left(I(a, r, s, p), x_{11}, \ldots, x_{1 a_{0}}\right)$ is self-radical. Since these ideals are self-radical and $\operatorname{rad}(I(a, r, s, p))$ is prime if $p=s_{h}$ for some $h$, the second statement follows immediately.

By the same method we see $I^{2}(a, r, s, p)$ is prime if $p=s_{h}$ for some $h, 0<h<$ $m$.

5. Calculation of dimension. In this section we use the fact that the $I\left(a, r, s, s_{h}\right)$ are prime to find a new generic point for them from which one can calculate their dimension.

For this, let $A_{k}$ be an $a_{k}-r_{k+1}$ by $r_{k}$ matrix of indeterminates and $B_{k}$ be the $a_{k}$ by $a_{k-1}$ matrix with $A_{k}$ in the top right-hand corner and zeroes elsewhere. Let $E_{k}^{\prime}$ be an $r_{k}$ by $a_{k-1}-r_{k}$ matrix of indeterminates and let $E_{k}$ be the $a_{k-1}$ by $a_{k-1}$ matrix with $E_{k}^{\prime}$ in the lower left-hand corner, ones down the diagonal and zeroes elsewhere. Clearly $\operatorname{det}\left(E_{k}\right)=1$. Let $U$ be an $\left(a_{1}-r_{2}\right)$ by $m$ matrix whose first $h$ rows are zero and let $U_{k}$ be the $k$ th column of $U$. Let $V_{k}$ be a $k$ by $\left(s_{k}-s_{k-1}-1\right)$ matrix of indeterminates and let

$$
W^{\prime}=U_{1} \#\left(\left.U\right|_{1}\right) V_{1} \# U_{2} \#\left(\left.U\right|_{2}\right) V_{2} \ldots\left(\left.U\right|_{m}\right) V_{m} \text {. }
$$

Then let $W$ be the $a_{1}$ by $a_{0}$ matrix with $W^{\prime}$ in the upper $a_{1}-r_{2}$ rows and zero elsewhere. 
Proposition 5.1. Let $K$ be a field, and let $I\left(a, r, s, s_{h}\right), W, E_{k}, B_{k}$ be as above. Then the K-homomorphism $f: K\left[X_{n}, \ldots, X_{1}\right] \rightarrow K\left[W, E_{k}, B_{k}\right]$ which takes

$$
\begin{aligned}
X_{n} & \rightarrow B_{n} E_{n}^{-1}, \\
X_{j} & \rightarrow E_{j+1} B_{j} E_{j}^{-1} \quad \text { for } 1<j<n, \\
X_{1} & \rightarrow E_{2} W
\end{aligned}
$$

is a generic point for $I\left(a, r, s, s_{h}\right)$; hence $\operatorname{ker} f=I\left(a, r, s, s_{h}\right)$. Moreover the fraction fields $K\left(B_{n} E_{n}^{-1}, E_{j} B_{j} E_{j-1}^{-1}, E_{1} W\right)$ and $K\left(A_{k}, E_{k}, U, V_{k}\right)$ are equal so that

$$
\begin{aligned}
\operatorname{dim} I\left(a, r, s, s_{h}\right)= & \sum_{k=2}^{n}\left(a_{k}-r_{k+1}\right) r_{k}+\sum_{k=2}^{n} r_{k}\left(a_{k-1}-r_{k}\right) \\
& +\left(a_{1}+a_{0}-r_{2}\right) m-\left(h+\frac{1}{2} m(m+1)+s_{0}+\ldots+s_{m-1}\right)
\end{aligned}
$$

where we set $r_{n+1}=0$.

Proof. By the construction of the $B_{j}$ and $W$ it is clear that $I\left(a, r, s, s_{h}\right) \subseteq \operatorname{ker}(f)$. To show the opposite inclusion it is enough to show the images of the $X_{i}$ in the fraction field of the domain $D=K\left[X_{n}, \ldots, X_{1}\right] / I\left(a, r, s, s_{h}\right)$ can be factored into the same form as the map $f$ takes. We do this as in the proof of Proposition 4.1, starting with $X_{n}$. It is easy to see by a specialization that $I_{r_{n}-1}\left(\bar{X}_{n}\right) \neq 0$ in $D$ where "-" denotes the image in $D$. Hence the last $r_{n}$ columns of $\bar{X}_{n}$ are linearly independent over $L=$ the fraction field of $D$. Since $I_{r_{n}}\left(\bar{X}_{n}\right)=0$, every other column of $\bar{X}_{n}$ is a linear combination of the last $r_{n}$ columns. Therefore there is a matrix $E_{n}^{\prime}$ over $L$ of the form of $E_{n}$ such that $\bar{X}_{n} E_{n}^{\prime}=B_{n}^{\prime}$ is a matrix with zeroes everywhere but the last $r_{n}$ columns. Hence $\bar{X}_{n}=B_{n}^{\prime}\left(E_{n}^{\prime}\right)^{-1}$ as required.

Consider the matrix $\left(E_{n}^{\prime}\right)^{-1} \bar{X}_{n-1}$. Since $\left(\bar{X}_{n} E_{n}^{\prime}\right)\left(E_{n}^{\prime-1} \bar{X}_{n-1}\right)=0$ and $\bar{X}_{n} E_{n}^{\prime}=B_{n}^{\prime}$ is a matrix whose last $r_{n}$ columns are linearly independent, the last $r_{n}$ rows of $\bar{X}_{n-1}^{\prime}=$ $\left(E_{n}^{\prime}\right)^{-1} \bar{X}_{n-1}$ must be zero. As above, since $I_{r_{n-1}}\left(\bar{X}_{n-1}^{\prime}\right)=I_{r_{n-1}}\left(\bar{X}_{n-1}\right)=0$, and since $\left(E_{n}^{\prime}\right)^{-1}$ does not affect the column space of $\bar{X}_{n-1}^{n}$, the last $r_{n-1}$ columns of $\bar{X}_{n-1}$ (and hence $\bar{X}_{n-1}^{\prime}$ ) are linearly independent and every column of $\bar{X}_{n-1}$ (and hence of $\left.\bar{X}_{n-1}^{\prime}\right)$ is a linear combination of the last $r_{n-1}$ columns. Therefore there exists a matrix $E_{n-1}^{\prime}$ of the form above such that $\bar{X}_{n-1}^{\prime} E_{n-1}^{\prime}=B_{n-1}^{\prime}$ is a matrix of the form of $B_{n-1}$. Thus

$$
\left(E_{n}^{\prime}\right)^{-1} \bar{X}_{n-1} E_{n-1}^{\prime}=B_{n-1}^{\prime} \text { and } \bar{X}_{n-1}=E_{n}^{\prime} B_{n-1}^{\prime}\left(E_{n-1}^{\prime}\right)^{-1} \text {. }
$$

By an easy induction we see we may choose $E_{j}^{\prime}, B_{j}^{\prime}$ of the form required such that $\bar{X}_{j}=E_{j+1}^{\prime} B_{j}^{\prime}\left(E_{j}^{\prime}\right)^{-1}$ for $1<j<n$ and such that $\left(E_{1}^{\prime}\right)^{-1} \bar{X}_{1}$ is a matrix $\bar{X}_{1}^{\prime}$ whose last $r_{2}$ rows are zero, and whose entries are algebraically independent; we now apply Proposition 33 of [15].

Proposition 5.1A [15]. Let $K$ be a field $s, p=s_{h}$ as above, and $W^{\prime}, U$, $V_{1}, \ldots, V_{m}$ as above. Then the $K$-homomorphism $g: K\left[\bar{X}_{1}^{\prime}\right] \rightarrow K\left[W^{\prime}\right]$ is a generic point for the ideal

$$
I=\sum I_{t}\left(\bar{X}_{1} \mid s_{t}\right)+\left(x_{11}, \ldots, x_{1 p}\right) \text {. }
$$

Moreover the fraction fields $K\left(W^{\prime}\right)$ and $K\left(U, V_{1}, \ldots, V_{m}\right)$ are equal so that

$$
\operatorname{dim} I=\left(a_{1}+a_{0}-r_{2}\right) m-\left(h+\frac{1}{2} m(m+1)+s_{0}+\ldots+s_{m-1}\right) .
$$


This shows we may factor $\bar{X}_{n-1}^{\prime}$ in the required form. To finish the proof it is enough to observe that $K\left(B_{n}\left(E_{n}\right)^{-1},\left(E_{n}\right)\left(B_{n-1} E_{n-1}^{-1}\right)\right)=K\left(B_{n}, E_{n}, B_{n-1} E_{n-1}^{-1}\right)$ since in $K\left(B_{n}\left(E_{n}\right)^{-1},\left(E_{n}\right)\left(B_{n-1} E_{n-1}^{-1}\right)\right)$ we have $K\left(B_{n}, B_{n-1}\right)$, and so may invert the minors of $B_{n}$ and the minors of $B_{n-1}$ and solve for the entries of $E_{n}$. Similarly

$$
K\left(B_{j} E_{j}^{-1}, E_{j}\left(B_{j-1} E_{j-1}^{-1}\right)\right)=K\left(B_{j}, E_{j},\left(B_{j-1} E_{j-1}^{-1}\right)\right),
$$

and so these observations together with Proposition 5.1A [15] show the second claim of Proposition 5.1. The dimension count is then trivial, using Proposition 5.1A [15].

COROLlARY 5.1. Suppose $\left(a_{n}, \ldots, a_{0}\right)$ is a sequence of nonnegative integers with $a_{n} \leqslant a_{n-1}$ and $\left(r_{n}, \ldots, r_{1}\right)$ and such that $r_{n}=a_{n}, r_{j}+r_{j-1}=a_{j-1}$ for $1<j<n$ and $r_{1} \leqslant a_{0}$. Let $s_{0}=0, s_{1}=1, \ldots, s_{r_{1}-1}=r_{1}-1, s_{r_{1}}=a_{0}$, and $p=0$. (So that these conditions give a complex with the appropriate rank conditions for exactness.) Then

$$
\operatorname{dim} I(a, r, s, 0)=\sum_{j=1}^{n} r_{j} a_{j-1} .
$$

Proof. By Proposition 5.1 this is immediate.

6. The perfection of $\mathrm{B}-\mathrm{E}(a)$. In this section we prove the perfection of the ideals $I\left(a, r, s, s_{h}\right)$ and further reduce the problem over a general ring to the generic case.

To this purpose we fix $a$ and $r$ and let $s=\left\{s_{0}, \ldots, s_{m}\right\}$ and $p=s_{h}$ vary, under the constraints of $\S 3$.

Proposition 6.1. Suppose $s_{h}<p<s_{h+1}$. Let $s^{\prime}=\left(s_{0}, \ldots, s_{h-1}, p, s_{h+1}, \ldots, s_{m}\right)$ and let $p^{\prime}=s_{h+1}$. Then

$$
I(a, r, s, p)=I\left(a, r, s^{\prime}, p\right) \cap I\left(a, r, s, p^{\prime}\right) .
$$

Proof. Certainly $I(a, r, s, p)$ is contained in the intersection. If we can show $I\left(a, r, s^{\prime}, p\right) I\left(a, r, s, p^{\prime}\right) \subseteq I(a, r, s, p)$ then since $\operatorname{rad}\left(I\left(a, r, s^{\prime}, p\right) I\left(a, r, s, p^{\prime}\right)\right)=$ $\operatorname{rad}\left(I\left(a, r, s^{\prime}, p\right) \cap I\left(a, r, s, p^{\prime}\right)\right)=I\left(a, r, s^{\prime}, p\right) \cap I\left(a, r, s, p^{\prime}\right)$ we will have proved the result. It suffices to show that for $p+1 \leqslant k \leqslant s_{h+1}$, if $M$ is any $h+1$ by $h+1$ minor of $\left(X_{1} \mid p\right), x_{1 k} M \in I(a, r, s, p)$. If the first row of $M$ coincides with the first row of $X_{1}$ this is obvious. If not, let $M^{*}$ be the unique $h+2$ by $h+2$ minor of $X_{1}$ which contains $M$ and $x_{1 k} . M^{*} \in I(a, r, s, p)$. The expansion by minors of $M^{*}$ along the first row is a sum containing $x_{1 k} M$ and all other terms are in $\left(x_{11}, \ldots, x_{1 p}\right)$. Hence $x_{1 k} M \in I(a, r, s, p)$ as required.

THEOREM 6.1. Let $K$ be any field, $R=K\left[X_{n}, \ldots, X_{1}\right]$. Suppose $m<a_{1}$ and $s_{h}<a_{0}$, and let $s, s^{\prime}, p^{\prime}$ be defined as above for any $s, p$. Then both $I\left(a, r, s, s_{h}\right)$ and $I\left(a, r, s, s_{h}+1\right)$ are perfect.

Proof. We use induction and Lemma 2.2A. Inducting on $\tilde{a}=\Sigma a_{i}$ allows us to assume the result for smaller matrices. Let $I(a, r, s, p)$ be maximal such that it is not perfect.

LEMMA 6.1A [21]. Let $K$ be a field and I a homogeneous ideal of the polynomial ring $K\left[x_{1}, \ldots, x_{n}\right]$. If $f$ is a form of positive degree not a zero-divisor on $I$, then $I$ is perfect if and only if $I+f R$ is perfect. 
If $p=s_{h}$ then by Theorem 4.1, I(a,r,s,p) is prime and so $x_{1 p+1}$ is not a zero-divisor on this ideal. But then the induction shows $I(a, r, s, p+1)$ is perfect and from Lemma 6.1A we conclude $I(a, r, s, p)$ is perfect.

Thus we may suppose $p=s_{h}+1$. By Proposition 6.1, $I(a, r, s, p)=I\left(a, r, s^{\prime}, p\right)$ $\cap I\left(a, r, s, p^{\prime}\right)$ where $s^{\prime}=\left\{s_{0}, \ldots, s_{h-1}, p, s_{h+1}, \ldots, s_{m}\right\}$ and $p^{\prime}=s_{h+1}$. By induction both of these ideals are perfect. We may compute their dimension from Proposition 5.1:

$$
\begin{aligned}
\operatorname{dim} I\left(a, r, s^{\prime}, p\right)= & \sum_{k=2}^{n} r_{k}\left(a_{k}+a_{k-1}-r_{k}-r_{k+1}\right)+\left(a_{1}+a_{0}-r_{2}\right) m \\
& -\left(h+\frac{1}{2} m(m+1)+s_{0}+\ldots+s_{h-1}+p+\ldots+s_{m-1}\right), \\
\operatorname{dim} I\left(a, r, s, p^{\prime}\right)= & \sum_{k=2}^{n} r_{k}\left(a_{k}+a_{k-1}-r_{k}-r_{k+1}\right)+\left(a_{1}+a_{0}-r_{2}\right) m \\
& -\left(h+1+\frac{1}{2} m(m+1)+s_{0}+\ldots+s_{h-1}+s_{h}+\ldots+s_{m-1}\right) .
\end{aligned}
$$

Since $p=s_{h}+1$, the dimensions (and hence the grades) of $I\left(a, r, s^{\prime}, p\right)$ and $I\left(a, r, s, p^{\prime}\right)$ are the same. Further, $I\left(a, r, s^{\prime}, p\right)+I\left(a, r, s, p^{\prime}\right)=I\left(a, r, s^{\prime}, p^{\prime}\right)$ and

$$
\begin{aligned}
\operatorname{dim} I\left(a, r, s^{\prime}, p^{\prime}\right)= & \sum_{k=2}^{n} r_{k}\left(a_{k}+a_{k-1}-r_{k}-r_{k+1}\right)+\left(a_{1}+a_{0}-r_{2}\right) m \\
& -\left(h+1+\frac{1}{2} m(m+1)+s_{0}+\ldots+s_{h-1}+p+\ldots+s_{h}\right)
\end{aligned}
$$

and so

$$
\text { grade } I\left(a, r, s^{\prime}, p\right)=\operatorname{grade} I\left(a, r, s, p^{\prime}\right)=\operatorname{grade} I\left(a, r, s^{\prime}, p^{\prime}\right)-1 .
$$

Since all these ideals are perfect Lemma 2.2A shows

$$
I(a, r, s, p)=I\left(a, r, s^{\prime}, p\right) \cap I\left(a, r, s, p^{\prime}\right)
$$

is also perfect which completes the proof of Theorem 6.1.

By the same token we note the ideals $I^{2}\left(a, r, s, s_{h}\right)$ are perfect, as either $I^{2}\left(a, r, s, s_{h}\right)=I\left(a, r, s, s_{h}\right)$ or $I^{2}\left(a, r, s, s_{h}\right)$ is given by a hypersurface on $R / I\left(a, r, s, s_{h}\right)$.

We now apply the notions of generically perfect [17] to deduce the general case of Theorem 6.1 from the generic case above.

LEMMA 6.2A [21]. If $I$ is a homogeneous ideal in $Z\left[x_{1}, \ldots, x_{n}\right]$ where $Z$ is the integers and the $x_{i}$ indeterminates, and $Z\left[x_{1}, \ldots, x_{n}\right] / I$ is $Z$-flat, then $I$ is strongly generically perfect of grade $g$ if and only if $K \otimes_{Z} I$ is perfect of grade $g$ in $K\left[x_{1}, \ldots, x_{n}\right]$ for every field $K$.

LemMA 6.3A [21]. Let I be a proper ideal of $Z\left[x_{1}, \ldots, x_{n}\right]$ such that $Z\left[x_{1}, \ldots, x_{n}\right] / I$ is $Z$-flat, $I$ is perfect and grade $I=g$. Suppose $R$ is a Noetherian ring and $a_{1}, \ldots, a_{n}$ are elements of $R$. Let $I^{\prime}$ be the ideal of $R$ given by the image of $I$ under the homomorphism of $Z\left[x_{1}, \ldots, x_{n}\right] \rightarrow R$ sending $x_{i}$ to $a_{i}$. Then grade $I^{\prime} \leqslant g$ and if grade $I^{\prime}=g$ then $I^{\prime}$ is a perfect ideal. 
TheOREM 6.2. Let $R$ be a commutative Noetherian ring and $M_{n}, \ldots, M_{1}$ matrices over $R$ with $M_{i}$ of size $a_{i} \times a_{i-1}$. Let $r=\left(r_{k}, \ldots, r_{1}\right), s=\left(s_{0}, \ldots, s_{m}\right)$ and $p=s_{h}$ be given with $m \leqslant r_{1}$. Let $I(a, r, s, p)(M)=I$ be the ideal of $R$ defined by the equations of $M_{i}$ corresponding to those above. Then

$$
\begin{aligned}
\operatorname{grade} I \leqslant & \sum_{k=2}^{n} r_{k}\left(a_{k}+a_{k-1}-r_{k}-r_{k+1}\right) \\
& +\left(a_{1}+a_{0}-r_{2}\right) m-\left(h+\frac{1}{2} m(m+1)+s_{0}+\ldots+s_{m-1}\right)
\end{aligned}
$$

and if equality occurs, then $I$ is perfect.

Proof. By Lemmas 6.2A and 6.3A and the computation of Theorem 6.1 (independent of the field $K$ ) we need only to show $R=$ $Z\left[X_{n}, \ldots, X_{1}\right] / I\left(a, r, s, s_{h}\right)$ is $Z$-flat. But this is if and only if $R$ is torsion free, i.e. if and only if $Z \cap I\left(a, r, s, s_{h}\right)=(0)$. This, however, is clear.

7. Normality. Although one can show the normality of the varieties $R / I\left(a, r, s, s_{h}\right)$, to avoid unnecessary complication we only do this for resolutions of length 2 which satisfy the rank condition of exactness.

THEOREM 7.1. Let $X$ be an $r \times n$ matrix of indeterminates and $Y$ an $n \times s$ matrix of indeterminates such that the entries of $X$ and $Y$ taken together are algebraically independent over $Z$, the integers. Suppose $r<n \leqslant r+s$. Let $P$ be the ideal of $R=Z[X, Y]$ generated by the entries of $X Y$ and $I_{n-r}(Y)$. Then $R / P$ is integrally closed.

Proof. First we show the theorem over an arbitrary field $K$. Let $S=K[X, Y] / I$ where $I$ is the ideal generated by the entries of $X Y$ plus the ideal $I_{n-r}(Y)$. By Theorem 6.1, $S$ is Cohen-Macaulay. Hence to show $S$ is integrally closed it is enough to show the codimension of the singular locus of $S$ is at least two. Let $J$ be the ideal in $K[X, Y]$ generated by the entries of $X Y$ plus $I_{n-r-1}(Y)$. Let $\bar{J}$ be the image of $J$ in $S$. We first show $\bar{J}$ is contained in the ideal defining the singular locus of $S$.

Let $\Delta$ be an $n-r$ by $n-r$ minor of $Y$. Without loss of generality, we may assume $\Delta$ is determined by the first $n-r$ rows and first $n-r$ columns, i.e. $\Delta=(1, \ldots, n-r \mid 1, \ldots, n-r)$. Suppose $i, j>n-r$. Then expanding the $n-r$ +1 by $n-r+1$ minor $(1, \ldots, n-r, i \mid 1, \ldots, n-r, j)$ we see that in $S_{\Delta}$, since this minor is zero, we may solve for $\bar{y}_{i j}$ in terms of the $y_{k m}$ with $k<n-r$ or $m \leqslant n-r$.

Now fix any row of $X$, say the $m$ th. In $S$,

$$
\begin{aligned}
& \bar{X}_{m 1} \bar{Y}_{11}+\ldots+\bar{X}_{m n} \bar{Y}_{n 1}=0 \\
& \vdots \\
& \bar{X}_{m 1} \bar{Y}_{1 n-r}+\ldots+\bar{X}_{m n} \bar{Y}_{n n-r}=0 ;
\end{aligned}
$$


choose $j, 1 \leqslant j \leqslant n-r$, and expand $\Delta$ along the $j$ th row $\bar{Y}_{j 1}, \ldots, \bar{Y}_{j n-r}$. Denote the $(n-r-1)$ by $(n-r-1)$ minor determined by

$$
(1, \ldots, \hat{i}, \ldots, n-r \mid 1, \ldots, \hat{j}, \ldots, n-r)
$$

by $\Delta_{i j}$. Then $\Delta=\sum_{i=1}^{n-r} \bar{Y}_{j i} \Delta_{j i}$ while

$$
\sum_{i=1}^{n-r} \bar{Y}_{k i} \Delta_{j i}=0
$$

if $1 \leqslant k \leqslant n-r$ and $k \neq j$.

Multiplying the $i$ th equation of (1) by $\Delta_{j i}$ and summing all the equations we obtain

$$
\sum_{i=1}^{n-r} \Delta_{j i}\left(\sum_{k=1}^{n} \bar{X}_{m k} \bar{Y}_{k i}\right)=0
$$

and so

$$
\sum_{k=1}^{n} \bar{X}_{m k}\left(\sum_{i=1}^{n-r} \bar{Y}_{k i} \Delta_{j i}\right)=0 .
$$

Consider the inner sum of (3). If $1 \leqslant k \leqslant n-r$ and $k \neq j$ this sum is zero by (2). If $k=j$ the inner sum gives $\Delta$. Hence

$$
\Delta \bar{X}_{m j} \in\left\langle\bar{X}_{m k} \mid k>n-r\right\rangle \text {. }
$$

Therefore, in $\left.S_{\Delta}, \bar{X}_{m j} \in\left\langle\bar{X}_{m k} \mid k\right\rangle n-r\right\rangle$, as $m$ was an arbitrary row of $\bar{X}$, i.e. $1 \leqslant m \leqslant r$. We see that in $S_{\Delta}, \bar{X}_{m j}$ for $1 \leqslant m \leqslant r, 1 \leqslant j \leqslant n-r$ can be solved for in terms of the $\bar{X}_{i k}$ with $k>n-r$.

Let $Z_{i j}$ be new indeterminates, $1 \leqslant i \leqslant r, n-r+1 \leqslant j \leqslant n$ and $t_{i j}$ be new indeterminates, where we let $1 \leqslant i \leqslant n, 1 \leqslant j \leqslant s$ but assume if $i, j>n-r$, then $t_{i j}=0$, i.e. we do not add a new indeterminate for these $i, j$. Let $T=K\left[Z_{i j}, t_{i j}\right]$ and map $T \stackrel{f}{\rightarrow} S$ by $Z_{i j}$ goes to $X_{i j}$ and $t_{i j}$ goes to $Y_{i j}$.

The discussion above shows if we let $\Delta^{\prime}=$ minor of the $t_{i j}$ given by $1<i<$ $n-r, 1 \leqslant j \leqslant n-r$ (so that $f\left(\Delta^{\prime}\right)=\Delta$ ) then $T_{\Delta^{\prime}} \rightarrow f^{\prime} \rightarrow S_{\Delta}$ is onto. As $T$ and $S$ are affine $K$-algebras, $\operatorname{dim} T=\operatorname{dim} T_{\Delta^{\prime}}$ and $\operatorname{dim} S=\operatorname{dim} S_{\Delta^{\prime}}$. The $\operatorname{dim} T$ is easy to compute; there are $r^{2} Z_{i j}$ and $(n-r) r+(n-r) s t_{i j}$. Hence

$$
\operatorname{dim} T=r n+s n-r s \text {. }
$$

We may calculate the $\operatorname{dim} S$ from Corollary 5.1 and we find $\operatorname{dim} S=r n+s n-$ $r s$. Since $f^{\prime}$ is an onto map and $T_{\Delta^{\prime}}$ is a domain of the same dimension as $f^{\prime}\left(T_{\Delta^{\prime}}\right)$, we see $f^{\prime}$ is an isomorphism. Thus $S_{\Delta}$ is regular.

Let us compute the height of the ideal $J$ generated by the entries of $X Y$ and those of $I_{n-r-1}(Y)$. This has a description (as in \$4) $a_{2}=r, a_{1}=n, a_{0}=s, r_{2}=r$, $r_{1}=n-r-1, s_{0}=0, \ldots, s_{n-r-2}=n-r-2, s_{n-r-1}=s, p=0$. By Proposition 5.1, we compute

$$
\mathrm{ht}(J)=r s+r+s-n+1 \text {. }
$$

Suppose $r+s>n$. Then $\operatorname{ht}(J) \geqslant \operatorname{ht}(I)+2$ and so, in $S$, ht $J S \geqslant 2$. By our remarks above, this forces $S$ to be integrally closed. 
Hence suppose $r+s=n$. Then we claim that any $r$ by $r$ minor $\Delta$ of $X$ is in the singular locus of $S$, i.e. $S_{\Delta}$ is regular. This easily follows from an argument similar to that above, observing $I_{n-r}(Y)=0$ in this case. The ideal $J$ generated by the entries of $X Y, I_{r-1}(X)$, and $I_{n-r-1}(Y)$ has a description, $a_{2}=r, a_{1}=n, a_{0}=s$, $r_{2}=r-1, r_{1}=n-r-1, s_{0}=0, \ldots, s_{n-r-2}=n-r-2, s_{n-r-1}=s$, and $p=$ 0 . A calculation using Proposition 5.1 shows $\mathrm{ht}(J)=r s+3$.

Hence $\operatorname{codim}(J S)=3$ and again $S$ is integrally closed.

To establish normality over $Z$, let $Q$ be the rationals. Then $Q[X, Y] / I$ is integrally closed and so if $S=Z[X, Y] / I$ is not integrally closed there is an element $f / c$ which is integral over $S$ with $f \in S$ and $c \in Z$. Since $S$ is faithfully flat over $Z,(c: f) \cap Z$ has grade at most one and is thus contained in a prime $p \in Z$. If we localize at $(p), r / c$ is integral over $Z_{(p)} \otimes_{Z} S$ but not in the ring. Hence we may assume $V[X, Y] / I$ is not integrally closed where $V$ is a discrete valuation ring. If $t$ is a parameter for $V$ we may assume there is an element $f / t$ which is integral over $V[X, Y] / I$. If $(f / t)^{n}=\sum_{k=1}^{n-1} v_{k}(f / t)^{k}$ is such an equation of integral dependence, then $t$ divides $f^{n}$. Thus it is enough to show $t S$ is prime. But $S / t S$ is a field $L$ and $L[X, Y] / I$ is a domain by Theorem 4.1.

8. Generic resolutions of length 2 . In this section we study the universal generic pair $(S, F)$ for resolutions of length two fixed Betti numbers. We refer the reader to the introduction, where the construction of this ring is carried through.

Throughout the rest of the section we fix generic matrices $X$ and $Y, X$ an $r$ by $n$ and $Y$ an $n$ by $s$ matrix where we assume $s, r<n \leqslant r+s$. If $\sigma$ is an $r$-element subset of $\langle 1, \ldots, n\rangle$, there is an associated $r$ by $r$ minor of $X, f_{\sigma}$, obtained by taking the columns corresponding to elements of $\sigma$. Of course $\sigma$ also determines a complementary $n-r$ element subset which we denote by $\sigma^{\prime}$. If $\tau$ is any $n-r$ element subset of $Y$, then $\sigma$ and $\tau$ determine an $n-r$ by $n-r$ minor of $Y$, which we denote $g_{\sigma, \tau}$, by choosing $n-r$ rows of $Y$ corresponding to elements of $\sigma^{\prime}$ and $n-r$ columns corresponding to $\tau$. Buchsbaum and Eisenbud showed

THEOREM [8]. Let $R$ be a commutative Noetherian ring and

$$
0 \rightarrow R^{r} \stackrel{A}{\rightarrow} R^{n} \stackrel{B}{\rightarrow} R^{s}
$$

an acyclic complex. Let $f_{\sigma}$ and $g_{\sigma, \tau}$ be defined as above for the matrices $A$ and $B$. Then there is a unique $c_{\tau} \in R$ such that for each $\sigma$ in $T(r, n)=$ the set of $r$-element subsets of $\langle 1, \ldots, n\rangle$,

$$
g_{\sigma, \tau}= \pm c_{\tau} f_{\sigma}
$$

The $c_{\tau}$ are called the multipliers and as one may assign an appropriate sign to the $f_{\sigma}$ we may assume (as we will henceforth do) that $g_{\sigma, \tau}=c_{\tau} f_{\sigma}$.

In general

$$
g_{\sigma, \tau} f_{\sigma^{\prime}}=g_{\sigma^{\prime}, \tau} f_{\sigma} .
$$

We now return to the generic case. Let $I$ be the ideal in $Z[X, Y]$ defined by the entries of $X Y$ and $I_{n-r}(Y) . I$ is clearly an ideal of Buchsbaum-Eisenbud type and Theorem 6.1 combined with Theorem 7.1 shows $R=Z[X, Y] / I$ is a CohenMacaulay normal domain. The equation (1) holds in $R$ and so $I_{r-1}(\bar{X}) \subseteq\left(f_{\sigma}: g_{\sigma, \tau}\right)$ 
for any fixed $\sigma$ and $\tau$, and hence grade $I_{r-1}(\bar{X})<1$. (Since $R$ is integrally closed this shows $h t\left(I_{r-1}(\bar{X})\right)=1$.)

However by the Buchsbaum-Eisenbud criteria for exactness (see the introduction) if the complex $0 \rightarrow R^{r} \stackrel{\bar{X}}{\rightarrow} R^{n} \stackrel{\bar{Y}}{\rightarrow} R^{s}$ is to be exact, $I_{r-1}(\bar{X})$ must have grade at least 2.

Let us consider the ring $S=R\left[g_{\sigma, \tau} / f_{\sigma}\right]$ where we let $\sigma$ be any element of $T(r, n)$ and $\tau$ any element of $T(n-r, s)$. (Of course from (1) we may assume $\sigma$ is fixed since $g_{\sigma^{\prime}, \tau} / f_{\sigma^{\prime}}=g_{\sigma, \tau} / f_{\sigma}$ for any $\sigma$ and $\sigma^{\prime}$ in $T(r, n)$.) As in Hochster [22] it is easy to see that if $J=I_{r-1}(\bar{X})$ then $\mathrm{ht}(J S) \geqslant 2$, and so if $S$ is either Cohen-Macaulay or integrally closed, grade $(J S) \geqslant 2$ and the complex

$$
\mathbf{F}: 0 \rightarrow S^{r} \stackrel{\bar{X}}{\rightarrow} S^{n} \stackrel{\bar{Y}}{\rightarrow} S^{s}
$$

is exact and $(S, \mathbf{F})$ give the required universal pair. (See the introduction.)

In characteristic zero, Hochster [22] showed the universal ring $S^{\prime}$ is the ring of invariants of a linear algebraic group acting linearly on a polynomial ring. The group is not, however, linearly reductive so that Theorem 1.1A does not seem to apply. However Hochster (conversation) indicated that the difficulty can be circumvented by standard invariant theory. Consequently in characteristic 0 , the universal generic ring $S^{\prime}$ is Cohen-Macaulay. Thus if we show $S$ is integrally closed and hence equal to $S^{\prime}, S$ will be Cohen-Macaulay (in characteristic zero).

We devote this section to a proof that $S$ is normal, relating its normality to the normality of the blow-up of a prime $p$ in $R$ and this to the reducedness of the special divisor.

Lemma 8.1 [4]. Let $R$ be an integrally closed Noetherian domain and $I$ be an ideal such that $\operatorname{gr}_{I}(R)$ is reduced. Then $R(I)=R+I t+I^{2} t^{2}+\cdots$, the Rees ring of $I$, is normal.

Proof. This is proved in [4] but we take time to note an elementary proof. Let $R_{-1}(I)=R(I)\left[t^{-1}\right]$ which we shall refer to as the extended Rees ring (although this is of ten called the Rees ring). As is well known and easy to see, $R_{-1}(I) /\left(t^{-1}\right) \simeq$ $\operatorname{gr}_{I}(R)$, and so $\left(t^{-1}\right)$ is reduced in $R_{-1}(I)$. In particular if $p$ is associated to $\left(t^{-1}\right)$, $R_{-1}(I)_{p}$ is a discrete valuation ring. We also see $R_{-1}(I)_{t^{-1}}=R\left[t, t^{-1}\right]$ which is integrally closed. However,

$$
R_{-1}(I)=R_{-1}(I)_{t^{-1}} \cap \bigcap_{p} R_{-1}(I)_{p}
$$

where the second intersection runs over all primes associated to $\left(t^{-1}\right)$. This shows $R_{-1}(I)$ is normal. Since $R[t]$ is normal and $R(I)=R_{-1}(I) \cap R[t]$ we see $R(I)$ is normal as required.

Proposition 8.1. Let $R$ be a Noetherian normal domain and $I=\left(a_{1}, \ldots, a_{n}, b\right)$ an ideal such that $\operatorname{gr}_{I}(R)$ is reduced. Then $R\left[a_{1} / b, \ldots, a_{n} / b\right]=S$ is normal.

Proof. Let $t$ be an indeterminate over $R$ and consider $S\left[b t,(b t)^{-1}\right]$. It is clear this is the same ring as $R\left[a_{1} t, \ldots, a_{n} t, b t,(b t)^{-1}\right]$ and this is just $R(I)_{b t}$. By Lemma 
8.1, $R(I)$ is normal and hence so is $R(I)_{b t}$. However, $b t$ is algebraically independent over $S$ and so $S\left[b t,(b t)^{-1}\right] \simeq S\left[t, t^{-1}\right]$ and this being normal implies $S$ is also.

We now return to our generic ring $R=Z[X, Y] /\left(X Y=0, I_{n-r}(Y)\right)$ and $S=$ $R\left[g_{\sigma, \tau} / f_{\sigma}\right]$. As we noted above we may assume $\sigma=\{n-r, \ldots, n\}$ and thus drop $\sigma$ from our notation.

By Proposition 8.2, to show $S$ is integrally closed it is enough to show the ideal $p=\left(f, g_{\tau}\right)_{\tau}$ satisfies the condition that its graded ring be reduced. The $g_{\tau}$ clearly run through the maximal minors of the first $n-r$ rows of $Y$.

We now place an order upon the $\tau$ in $T=T(n-r, s)$ by saying $\left(i_{1}, \ldots, i_{n-r}\right)$ $\leqslant\left(j_{1}, \ldots, j_{n-r}\right)$ if and only if

$$
i_{1} \leqslant j_{1}, \ldots, i_{n-r} \leqslant j_{n-r} .
$$

$T$ thus becomes a partially ordered set and we define an ideal $H \subset T$ to be any subset with the property that $\sigma \in H$ and $\sigma^{\prime}<\sigma$ imply $\sigma^{\prime} \in H$.

In a similar way if $Y$ is an $n$ by $s$ matrix we may place partial order on all the minors of $Y$. Any $k$ of $k$ minor of $Y$ is determined by $k$ rows $i_{1}<\ldots<i_{k}$ and $k$ columns, $j_{1}<\ldots<j_{k}$, and we denote this minor by $\left(i_{1}, \ldots, i_{k} \mid j_{1}, \ldots, j_{k}\right)$. We say

$$
\left(i_{1}, \ldots, i_{k} \mid j_{1}, \ldots, j_{k}\right) \leqslant\left(i_{1}^{\prime}, \ldots, i_{m}^{\prime} \mid j_{1}^{\prime}, \ldots, j_{m}^{\prime}\right)
$$

if and only if both $k \geqslant m$ and $i_{1} \leqslant i_{1}^{\prime}, \ldots, i_{m} \leqslant i_{m}^{\prime}$, and $j_{1} \leqslant j_{1}^{\prime}, \ldots, j_{k} \leqslant j_{k}^{\prime}$. (See [12], [13], or [14].) This order clearly agrees with that established above for the $g_{\tau}$. We define an ideal in the same way. Let $G$ be the partially ordered set defined by the above relation, and if $\sigma \in G$, let $\bar{\sigma}$ be the corresponding minor.

LEMMA 8.2 [13]. Let $Y$ be a generic $n$ by $s$ matrix over $Z$, the integers. Let $G$ be as above and suppose $J \subset G$ is an ideal of $G$ and $\sigma=\left(i_{1}, \ldots, i_{k} \mid j_{1}, \ldots, j_{k}\right)$ is an element which is minimal among elements of $G$ not in $J$. If $J^{\prime} \subset G$ is the set of all $\beta \in G$ such that $\beta \neq \sigma$, then $(\bar{J}: \bar{\sigma})=\bar{J}^{\prime}$.

Corollary 8.1. Let $Y$ be as above and let $H \subset T$ be an ideal, where $T=$ $T(n-r, s)$ is the subset of $G$ determined by all elements of the form $\left(1, \ldots, n-r \mid i_{1}, \ldots, i_{n-r}\right)$. Let $J=H \cup\left\{\left(i_{1}, \ldots, i_{t} \mid j_{1}, \ldots, j_{t}\right) \mid t>n-r+1\right\}$. Finally, suppose $\sigma=\left(1, \ldots, n-r \mid i_{1}, \ldots, i_{n-r}\right)$ is an element of $T$ which is minimal above J. Then $(\bar{J}: \bar{\sigma})=I\left(i_{1}, \ldots, i_{n-r}\right)$ where $I\left(i_{1}, \ldots, i_{n-r}\right)$ is the ideal defined by $\sum_{j=1}^{n-r} I_{j-1}\left(Y \mid i_{j}-1\right)+I_{n-r}(Y)$.

Proof. From the partial order on $G$ it is easy to see $J$ is an ideal. By Lemma $8.2(\bar{J}: \bar{\sigma})$ is the set of all minors $\beta \in G$ such that $\beta \neq \sigma$. Let us first show $I_{t-1}\left(Y \mid i_{t}-1\right)$ is in this set. Any $t$ by $t$ minor $\bar{\alpha}$ in $I_{t-1}\left(Y \mid i_{t}-1\right)$ is given by $\left(j_{1}, \ldots, j_{t} \mid m_{1}, \ldots, m_{t}\right)$ where $m_{t}<i_{t}$. This shows $\alpha \ngtr \sigma$ and so $\bar{\alpha} \in(\bar{J}: \bar{\sigma})$. Since $I_{n-r}(Y)$ is contained in $\bar{J}$ we see $I\left(i_{1}, \ldots, i_{n-r}\right) \subseteq(\bar{J}: \bar{\sigma})$.

If $\alpha=\left(j_{1}, \ldots, j_{t} \mid m_{1}, \ldots, m_{t}\right) \in(\bar{J}: \bar{\sigma})$ then, by Lemma 8.2, $\alpha \ngtr \sigma$ and so since we may assume $t \leqslant n-r$, and either some $j_{k}<k$ or some $m_{k}<i_{k}$. The former cannot occur as $0<j_{1}<\ldots<j_{k}$. Thus the latter occurs and by expanding $\bar{\alpha}$ around its $t \times t$ minors we see that $\bar{\alpha} \in I_{t-1}\left(Y \mid i_{k}\right)$. This finishes the proof of Corollary 8.2 . 
Notice that the ideal $I\left(i_{1}, \ldots, i_{n-r}\right)$ has a description (as above) by $s_{0}=i_{1}-1$, $s_{1}=i_{2}-1, s_{n-r-1}=i_{n-r}-1, s_{n-r}=s$.

LEMMA 8.3. Let $X$ and $Y$ be generic $r$ by $n$ and $n \times s$ matrices respectively, with $r<n \leqslant r+s$. Let $T=T(n-r, s)$ be the partially ordered set above and suppose $H \subset T$ is an ideal. Let $\sigma \in T$ be an element minimal over $H$. Let $I$ be the ideal generated by the entries of $X Y$, the $r$ by $r$ minor of $X$ determined by its last $r$ columns and $I_{n-r}(Y)$. Then $(I, \bar{H}: \bar{\sigma})$ is a prime ideal and $\bar{\sigma}$ is not a zero-divisor module in it.

Proof. The ideal $(I, \bar{H}: \bar{\sigma})$ certainly contains $I+(\bar{H}: \bar{\sigma})$ which by Corollary 8.2 is equal to $I+I\left(i_{1}, \ldots, i_{n-r}\right)$ where $\sigma=\left(1, \ldots, n-r \mid i_{1}, \ldots, i_{n-r}\right)$. However this ideal is an ideal in the second principal radical system of $\$ 4$ and is prime by Theorem 4.1. The last statement follows as $\bar{\sigma}$ is not in the ideal.

Recall if $H$ is a finite poset and $\left\{x_{\alpha}\right\} \subseteq R$ is a set of elements in a commutative ring $R$ indexed by $H$, we say an ideal $I$ of $R$ is an $H$ ideal if $J=\bar{I}$ for some ideal $I$ of $H$. If $J$ is an ideal we set $J^{*}$ equal to the ideal generated by all $x_{\tau} \in J$.

Definition 8.1 [28]. (See the introduction.) Let $R, H,\left\{x_{\alpha}\right\}$ be as above. The $\left\{x_{\alpha}\right\}$ are said to be a weak $d$-sequence if whenever $I$ is an $H$ ideal containing all $x_{\beta}$ where $\beta<\alpha$ but not containing $x_{\alpha}$ then

(1) $\left(I: x_{\alpha}\right) \cap \bar{H}=\left(I: x_{\alpha}\right)^{*}$ is an $H$ ideal.

(2) If $x_{\alpha} \in\left(I: x_{\alpha}\right)$ then $x_{\alpha} x_{\beta} \in I \bar{H}$.

(3) If $x_{\alpha} \notin\left(I: x_{\alpha}\right)$ then $\left(I: x_{\alpha}\right)=\left(I: x_{\alpha}^{2}\right)$.

TheOREM 8.1. Let $X, Y$ be generic matrices as above and let $R=Z[X, Y] / J$ where $Z$ is the integers and $J$ is the ideal generated by all the entries of $X Y$ and $I_{n-r}(Y)$. Let $H$ be the poset of maximal minors of the top $n-r$ rows of $Y$. If $\alpha \in H$, let $\bar{\alpha}$ be the corresponding minor. Let $H^{+}$be $H \cup\{0\}$ and let $\overline{0}=$ the $r \times r$ minor $\Delta$ of $X$ determined by the last $r$ columns of $X$. (Here $0<\alpha$ for all $\alpha \in H$.) Then $\left\{\bar{\alpha} \mid \alpha \in H^{+}\right\}$is a weak d-sequence.

Proof. Let $I$ be as in Definition 8.1. Property (3) is immediate, since each $(I: \bar{\alpha})$ is prime by Lemma 8.3 and $\bar{\alpha} \notin(I: \bar{\alpha})$ so that $(I: \bar{\alpha})=\left(I: \bar{\alpha}^{2}\right)$. If $\bar{\beta} \in(I: \bar{\alpha})$ then again by Lemma $8.3, \bar{\beta} \neq \bar{\alpha}$ and then the standard quadratic relation (see [12], [13], or [25]) shows $\bar{\beta} \bar{\alpha} \in I \bar{H}$.

Finally, $(I: \bar{\alpha}) \cap \bar{H}^{+}=\langle\bar{\beta} \mid \beta \neq \alpha\rangle+I=(I: \bar{\alpha})$ and this is an $H$ ideal since $\langle\beta \mid \beta \neq \alpha\rangle$ is an ideal.

Recall if $\left\{x_{\alpha}\right\}$ is weak $d$-sequence an ideal $J$ is said to be related to $\left\{x_{\alpha}\right\}$ if $J$ is of the form $\left(I: x_{\alpha}\right)+\bar{H}$ where $I$ is an $H$ ideal containing all $x_{\beta}$ with $\beta<\alpha$ but not $x_{\alpha}$. The related ideals of the weak $d$-sequence above are given by Lemma 8.3 ; by Theorem 6.1 all of them are perfect and prime. Let $\alpha=(1, \ldots, n-r \mid s-n+$ $r, \ldots, s)$, and let $I$ be the ideal generated by all $\bar{\beta}, \beta \in H^{+}-\{\alpha\}$. Then by Lemma 8.3, $(I: \bar{\alpha})$ is the ideal defined in $R$ by the vanishing of all $y_{i j}$ with $j<s+r-n$. This ideal clearly contains all other related ideals. We recall, (see the introduction)

THEOREM 8.2 [28]. Suppose $R$ is a commutative ring and $\left\{x_{\alpha} \mid \alpha \in H\right\}$ is a weak $d$-sequence. Suppose the ideal I generated by all the $x_{\alpha}$ is prime and $I_{q}^{(n)}=I_{q}^{n}$ for all primes $q$ associated to any related ideal. Then $I^{(n)}=I^{n}$. 
TheOREM 8.3. Let $R$ be as in Theorem 8.1 and let $p=\left\langle\bar{\alpha} \mid \alpha \in H^{+}\right\rangle$. Then $\operatorname{gr}_{p}(R)$ is a domain and consequently the ring $R\left[g_{\tau} / f\right]$ described above is integrally closed.

Proof. The last statement follows from Proposition 8.2 and the first statement. As $p$ is prime (see [26]), to show $\operatorname{gr}_{p}(R)$ is a domain it is enough to show $R_{p}$ is regular and $p^{(n)}=p^{n}$ for all $n$.

Let $Q$ be the maximal related ideal defined by all $y_{i j}$ with $j<s+r-n$ plus $(1, \ldots, n-r \mid r+s-n, \ldots, s)$ and $\Delta$. Since $Q$ is prime, if we show $R_{Q}$ is regular and $p_{Q}$ to be a complete intersection then $p_{Q}^{(n)}=p_{Q}^{n}$ for all $n$. But any other prime associated to a related ideal is equal to a related ideal (all related ideals are prime) and hence contained in $Q$. Thus if $Q^{\prime}$ is related $p_{Q^{\prime}}^{(n)}=p_{Q^{\prime}}^{n}$ and Theorem 8.2 shows that $p^{(n)}=p^{n}$ for all $n$. However, $p^{(n)}=p^{n}$ for all $n$ and $R_{p}$ regular imply $\operatorname{gr}_{p}(R)$ is a domain.

Let us consider $R_{Q}$. The ideal $J$ defining $R$ are the equations $X Y=0$ and $J_{n-r}(Y)=0$. It is easy to see that any $r$ by $r$ minor of $X$ is in the singular locus of $Z[X, Y] / J=R$. Certainly some $r \times r$ minor of $X$ is not in $Q$, say $w=$ $(1, \ldots, r \mid 1, \ldots, r)$ and so $R_{Q}$ is regular. When we invert $w$, the ideal $p$ is generated by $\Delta$ since the relation $f_{\sigma} g_{\bar{\sigma}, \tau}=f_{\bar{\sigma}} g_{\sigma, \tau}$ shows that for all $\tau$, if we invert some $f_{\bar{\sigma}}$, then $\left\langle g_{\sigma, \tau}\right\rangle \subseteq\left(f_{\sigma}\right)$. Hence in $R_{Q}, p_{Q}$ becomes a complete intersection and this shows $p_{Q}^{(n)}=p_{Q}^{n}$ for all $n$. Thus $p^{(n)}=p^{n}$ for all $n$ as required.

\section{BIBLIOGRAPHY}

1. S. Abeasis and A. Del Fra, Young diagrams and ideals of Pfaffians, preprint, Universita Degli Studi di Roma, 1979-1979.

2. M. Auslander and D. Buchsbaum, Codimension and multiplicity, Ann. of Math. 68 (1958), 625-657.

3. S. Barger, Generic perfection and the theory of grade, Thesis, Univ. of Minnesota, 1970.

4. J. Barshay, Graded algebras of powers of ideals generated by A-sequences, J. Algebra 25 (1973), 90-99.

5. Boutot, Pure subrings of rings with rational singularities have rational singularities, preprint.

6. M. Brodmann, The asymptotic nature of the analytic spread, preprint.

7. D. Buchsbaum and D. Eisenbud, What makes a complex exact?, J. Algebra 25 (1973), 259-268.

8. __ Some structure theorems for finite free resolutions, Advances in Math. 12 (1974), 84-139.

9. D. Buchsbaum and D. S. Rim, A generalized Koszul complex. II. Depth and multiplicity, Trans. Amer. Math. Soc. 111 (1964), 197-224.

10. L. Burch, Codimension and analytic spread, Proc. Cambridge Philos. Soc. 72 (1972), 369-373.

11. R. Cowsik and M. Nori, On the fibers of blowing up, preprint.

12. C. De Concini and C. Procesi, A characteristic free approach to invariant theory, Advances in Math. 21 (1976), 333-354.

13. C. De Concini, D. Eisenbud and C. Procesi, Algebras with straightening law (in preparation).

14. __ Young diagrams and determinantal ideals, preprint.

15. J. Eagon and M. Hochster, Cohen-Macaulay rings, invariant theory, and the generic perfection of determinantal loci, Amer. J. Math. 93 (1971), 1020-1058.

16. J. Eagon and D. Northcott, Ideals defined by matrices and a certain complex associated with them, Proc. Roy. Soc. London Ser. A 269 (1962), 188-204.

17. , Generically acyclic complexes and generically perfect ideals, Proc. Roy. Soc. London Ser. A 299 (1967), 147-172.

18. D. Eisenbud and C. Huneke, On the Cohen-Macaulayness and torsion freeness of $\mathrm{gr}_{I}(R)$, preprint.

19. T. Gulliksen and $O$. Negard, Un complexe résolvent pour certains idéaux déterminentiels, C. $R$. Acad. Sci. Paris Ser. A 274 (1972), A16-A19.

20. M. Hochster, Generically perfect modules are strongly generically perfect, Proc. London Math. Soc. 23 (1971), 477-488. 
21. Grassmannians and their Schubert subvarieties are arithmetically Cohen-Macaulay, J. Algebra 25 (1973), 40-57.

22. __ Topics in the homological theory of modules over commutative rings, C.B.M.S. Regional Conf. Ser. in Math., no. 24, Amer. Math. Soc., Providence, R.I., 1975.

23. _ Criteria for equality of ordinary and symbolic powers of primes, Math. Z. 133 (1973), 53-65. 24. M. Hochster and J. L. Roberts, Rings of invariants of reductive groups acting on regular rings are Cohen-Macaulay, Advances in Math. 13 (1974), 115-175.

25. W. V. D. Hodge and D. Pedoe, Methods of algebraic geometry, vol. 2, Cambridge Univ. Press, Cambridge, Mass., 1968.

26. C. Huneke, Determinantal ideals and questions related to factoriality, Thesis, Yale Univ., 1978.

27. , On the symmetric and Rees algebras of an ideal generated by a d-sequence, J. Algebra 62 (1980), 267-275.

28. __ Powers of ideals generated by weak d-sequences, J. Algebra (to appear).

29. The theory of $d$-sequences and powers of ideals, Advances in Math. (to appear).

30. __ Symbolic powers of primes and special graded algebras (to appear).

31. J. Igusa, On the arithmetic normality of the Grassmann variety, Proc. Nat. Acad. Sci. U.S.A. 40 (1954), 309-313.

32. G. Kempf, On the collapsing of homogeneous bundles, Invent. Math. 37 (1976), 229-239.

33. , Images of homogeneous vector bundles and varieties of complexes, Bull. Amer. Math. Soc. 81 (1975), 900-901.

34. H. Kleppe and D. Laksov, The algebraic structure and deformation of Pfaffian schemes, Institute Mittag-Leffler, Report No. 3, 1979.

35. R. Kutz, Cohen-Macaulay rings and ideal theory in rings of invariants of algebraic groups, Trans. Amer. Math. Soc. 194 (1974), 115-129.

36. D. Laksov, The arithmetic Cohen-Macaulay character of Schubert schemes, Acta Math. 129 (1972), 1-9.

37. A. Lascoux, Syzygies des variétés déterminantales, Advances in Math. 30 (1978), $202-237$.

38. H. Matsumura, Commutative algebra, Benjamin, New York, 1970.

39. C. Musili, Postulation formula for Schubert varieties, J. Indian Math. Soc. 36 (1972), 143-171.

40. D. G. Northcott, Additional properties of generically acyclic complexes, Quart. J. Math. Oxford Ser. 20 (1969), 65-80.

41. __ Grade sensitivity and generic perfection, Proc. London Math. Soc. 20 (1970), 597-618.

42. D. G. Northcott and D. Rees, Reductions of ideals in local rings, Proc. Cambridge Philos. Soc. 50 (1954), 145-158.

43. C. Peskine and L. Szpiro, Dimension projectif finie et cohomologie locale, Inst. Hautes Étude Sci. Publ. Math. 42 (1973), 323-395.

44. L. Robbiano and G. Valla, Primary powers of a prime ideal, Pacific J. Math. 63 (1976), $491-498$.

45. , On normal flatness and normal torsion freeness, J. Algebra 43 (1976), 222-229.

46. D. Sharpe, On certain polynomial ideals defined by matrices, Quart. J. Math. Oxford Ser. 15 (1964), 155-175.

Department of Mathematics, University of Michigan, Ann Arbor, Michigan 48109 